hep-th/0001066

TIFR/TH/00-02

KCL-TH-00-02

\title{
Brane-Antibrane Constructions
}

\author{
Sunil Mukhi ${ }^{1}$, Nemani V. Suryanarayana ${ }^{1}$ and David Tong ${ }^{2}$ \\ 1 Tata Institute of Fundamental Research, \\ Homi Bhabha Rd, Mumbai 400 005, India \\ ${ }^{2}$ Department of Mathematics, Kings College, \\ The Strand, London, WC2R 2LS, UK
}

\begin{abstract}
In type II string theories, we examine intersecting brane constructions containing brane-antibrane pairs suspended between 5-branes, and more general non-BPS constructions. The tree-level spectra are obtained in each case. We identify various models with distinct physics: parallel brane-antibrane pairs, adjacent pairs, non-adjacent pairs, and configurations which break all supersymmetry even though any pair of branes preserves some supersymmetry. In each case we examine the possible decay modes. Some of these configurations turn out to be tachyon-free, stable non-BPS states. We use T-duality to map some of our brane constructions to brane-antibrane pairs at ALE singularities. This enables us to explicitly derive the spectra by the analogue of the quiver construction, and to compute the sign of the brane-antibrane force in each case.
\end{abstract}

January 2000

E-mail: mukhi@tifr.res.in, nemani@tifr.res.in, tong@mth.kcl.ac.uk 


\section{Introduction}

The study of unstable branes in type II superstring theories has made considerable progress over the last two years [1]. The relevant unstable branes are of two types: $\mathrm{D} p$ brane-antibrane pairs, ( $p$ even for type IIA, odd for type IIB) and unstable $\mathrm{D} p$-branes with $p$ odd for type IIA, even for type IIB. In both cases, the instability is signalled by the presence of a tachyon, and it is possible to identify a variety of decay modes. These assemble themselves into interesting sequences that terminate with stable BPS D-branes.

The unstable branes and their decay modes form a beautiful and fundamental structure, which has been interpreted in terms of K-theory[2, 3]. This structure can then be used to study more complicated situations such as orientifolds, orbifolds and Calabi-Yau compactifications. In these cases it often happens that one discovers novel stable non-BPS states (see Refs. [1],4], [0] and references therein).

In the present paper, we attempt to generalise these elegant discoveries to situations where unstable D-branes are suspended between other branes. As we will see, along with many phenomena that are familiar from the study of infinite or toroidally compactified unstable branes, there are also new constraints and novel physical situations that have no counterpart in the simpler models.

All the brane constructions that we study will have completely broken supersymmetry. However, they are states of type II string theories, and therefore are endowed with special properties that arise from the fact that the underlying theory is supersymmetric. One interesting class of models that we define has broken supersymmetry despite the absence of brane-antibrane pairs or single unstable branes. In these models one arranges at least three different types of branes together, in such a way that each pair preserves some supersymmetry, but all the branes together break all supersymmetry.

Even in supersymmetric models, the study of suspended branes suffers from some uncertainties, as it is often hard to reliably extract the spectrum of light states. These uncertainties are all the more severe in the present case, as the brane configurations lack supersymmetry which would have classified the possible states into multiplets. As most of the models we study involve D-branes suspended between NS5-branes, we will find it useful to dualise away the NS5-branes into ALE geometry[6] and then study the model using quiver techniques[7], where many of the relevant quantities can in fact be reliably computed.

Although this paper will deal for the most part with unstable configurations, it is meaningful to study their spectra at weak coupling by working at string tree-level. Here 
one can identify the presence or absence of potential decay modes related to tachyon condensation, even though the configuration actually gets destabilised after loop effects are taken into account. All discussions of unstable configurations should be understood in the light of this comment.

The plan of this paper is as follows. In Section 2, we describe systems of parallel D-brane-antibrane pairs suspended between NS5-branes and D5-branes. These are closest in behaviour to the noncompact parallel brane-antibrane pairs, though we identify some differences that arise due to boundary conditions at the ends of the D-branes. In Section 3 we look at unstable uncharged D-branes suspended between NS5-branes and examine some possible decay modes. In Section 4 we consider systems of parallel NS5-branes with a Dbrane stretched across one segment and a $\overline{\mathrm{D}}$-brane stretched across the adjacent segment. In this case the pair cannot annihilate. We examine some related models and argue that in general these pairs will repel each other. We also consider configurations with more NS5-branes, where the brane-antibrane pair is non-adjacent. In Section 5 we introduce the "Borromean branes", configurations of branes in which supersymmetry is broken by all the branes together but not by any given pair of branes. This has the interesting consequence that there is no perturbative tachyon, and the configuration can potentially be stable. In Section 6 we describe the duality which relates suspended branes between NS5-branes to fractional branes at ALE singularities. Although this duality has been described and used before in the literature, we give a slightly different and very explicit derivation, which will hopefully make it somewhat clearer. In Section 7 we apply this duality to analyse parallel and adjacent brane-antibrane pairs from the point of view of quiver theory. In Section 8 we compute the spectra of open strings in these configurations by constructing boundary states for the relevant fractional branes. We also use this formalism to compute the forces between different pairs of fractional branes, confirming some of the speculations made in earlier sections.

We will not provide a review of various fundamental aspects of brane-antibrane dynamics that will be made use of in this paper. For this, the reader is advised to consult Refs. [1, 4], 5$]$.

\section{Suspended Parallel Brane-antibrane Pairs}

Consider, in type IIA string theory, a model with a pair of NS5-branes extending along the directions $\left(x^{1}, x^{2}, x^{3}, x^{4}, x^{5}\right)$, and located at $\left(x^{6}, x^{7}, x^{8}, x^{9}\right)=(0,0,0,0)$ and 
$\left(L_{6}, 0,0,0\right)$ respectively. Thus they are parallel and separated by a finite distance $L_{6}$ along $x^{6}$. Between these, we suspend a D4-brane and a $\bar{D} 4$-brane along the $x^{6}$ direction. They extend along the directions $\left(x^{1}, x^{2}, x^{3}\right)$ and can be separated from each other along $\left(x^{4}, x^{5}\right)$ (Fig.(2.1)). Without the antibrane, this model would belong to the class of brane constructions analysed in Ref. [8].

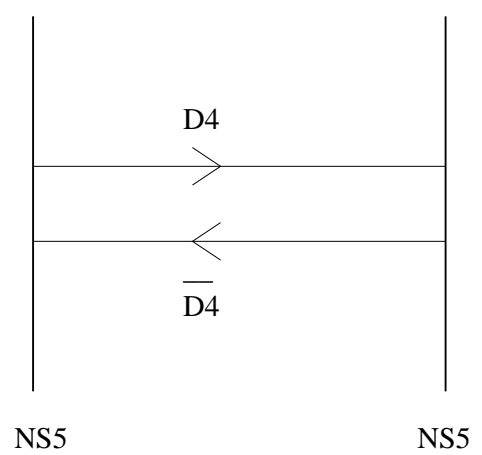

Fig.(2.1): A D4 - $\overline{\mathrm{D}} 4$ pair suspended between parallel NS5-branes in type IIA.

We expect that the common 3+1-dimensional world-volume supports a non-supersymmetric field theory. The spectrum of light states, including possible tachyons, can be deduced as follows. First consider an infinitely extended D4- $\overline{\mathrm{D}} 4$ pair. As is well-known [1], the openstring states have four sectors, corresponding to the Chan-Paton factors:

$$
a=\left(\begin{array}{ll}
1 & 0 \\
0 & 0
\end{array}\right), \quad b=\left(\begin{array}{ll}
0 & 0 \\
0 & 1
\end{array}\right), \quad c=\left(\begin{array}{ll}
0 & 1 \\
0 & 0
\end{array}\right), \quad d=\left(\begin{array}{ll}
0 & 0 \\
1 & 0
\end{array}\right)
$$

The first two sectors come from strings beginning and ending on the same brane(antibrane). Together, these give a GSO-projected spectrum of $U(1) \times U(1)$ vector multiplets, containing two 4+1-dimensional gauge fields $A_{\mu}^{(I)}$, two sets of five massless scalar fields $X_{i}^{(I)}$ and two sets of 4 massless Majorana fermions $\chi_{A}^{(I)}$, where $i=1, \ldots, 5, A=1, \ldots, 4$ and $I=1,2$. The index $I$ here labels the Chan-Paton factors $a, b$. In the other two sectors, associated to open strings going from the brane to the antibrane and vice-versa, there is a complex tachyon $T$ carrying charges $(1,-1)$ under $U(1) \times U(1)$, and another set of massless Majorana fermions $\lambda_{A}^{(I)}$. (This time the index $I$ labels the Chan-Paton factors $c, d$.) These correspond to "anti-GSO" states that arise because of the fact that the two branes carry opposite RR charges.

Together, the infinite brane-antibrane pair breaks all 32 supersymmetries of the bulk theory. Hence we expect to find 32 massless Goldstone fermions ("goldstinoes") on the world-volume of this pair. Actually we have 64 massless fermions, from the $\chi_{A}^{(I)}$ and $\lambda_{A}^{(I)}$. 
One way to identify which of these are goldstinoes is to use the fact that when we quotient type IIA string theory, with a D4 - $\overline{\mathrm{D}} 4$ pair, by the symmetry $(-1)^{F_{L}}$, we end up with type IIB string theory with an unstable D4-brane in it. This unstable brane carries a real tachyon and precisely 32 massless Majorana fermions. In this case it is clear that all the fermions must be goldstinoes.

It follows that in the $\mathrm{D} 4-\overline{\mathrm{D}} 4$ pair, the goldstinoes must be those fermions which survive the $(-1)^{F_{L}}$ quotient $\left((-1)^{F_{L}}\right.$ has no twisted sectors on open-string states, so those fermions which survive the quotient and are goldstinoes in the final theory must have been goldstinoes before taking the quotient too). This quotient acts on the Chan-Paton factors as conjugation by $\sigma_{1}$, hence the surviving Chan-Paton factors on the unstable D4-brane are $1, \sigma_{1}$. It follows that $\chi_{A}^{+}=\chi_{A}^{(1)}+\chi_{A}^{(2)}$ and $\lambda_{A}^{+}=\lambda_{A}^{(1)}+\lambda_{A}^{(2)}$ are the goldstinoes on the $\mathrm{D} 4-\overline{\mathrm{D}} 4$ pair.

Now let us go back to the model of a $\mathrm{D} 4-\overline{\mathrm{D}} 4$ pair suspended between NS5-branes and work out the spectrum. Again we have a $U(1) \times U(1)$ gauge theory, whose spectrum is a truncation of that on the infinite $\mathrm{D} 4-\overline{\mathrm{D}} 4$ pair. The truncation arises from the boundary conditions at the two ends of the 4-branes.

For the massless fields in the GSO-projected sector (associated to Chan-Paton factors $\left.1, \sigma_{3}\right)$ the truncation is well-known: the massless scalars that are collective coordinates for broken translation invariance are projected out whenever they correspond to directions along which the 4-branes cannot move. Since the NS5-branes on which they end are located at fixed values of $x^{7}, x^{8}, x^{9}$, it follows that the scalars $X_{7}^{(I)}, X_{8}^{(I)}, X_{9}^{(I)}$ are projected out. Along with these, the gauge field component $A_{6}^{(I)}$ and half the associated fermions, say $\chi_{3}^{(I)}, \chi_{4}^{(I)}$ are projected out. The result is a set of massless fields: $A_{\mu}^{(I)}, X_{i}^{(I)}, \chi_{A}^{(I)}$ where $\mu=0, \ldots, 3, i=4,5$ and $A=1,2$. This is a pair of vector multiplets of $\mathcal{N}=2$ supersymmetry in $3+1$ dimensions.

In the anti-GSO sector, supersymmetry is clearly not available to guide us in finding the spectrum that survives. We shall work in the limit in which the separation between NS5-branes is very much smaller than the string length scale, ensuring that the higher tachyon Kaluza-Klein modes have positive mass-squared and so, as for other fields, we restrict attention to the constant modes. It is plausible, and we will confirm this later, that the complex tachyon survives the boundary conditions. Recall that for an infinite D4 - $\overline{\mathrm{D}} 4$ pair, the tachyon is associated to the NS sector ground state for open strings stretching between the brane and the antibrane. Thus we are claiming that this ground state is not projected out by the boundary conditions. It is physically evident that the 
D4- $\overline{\mathrm{D}} 4$ pair suspended between parallel NS5-branes should be unstable, just as an infinite, or wrapped, D4 - $\overline{\mathrm{D}} 4$ pair would be. Hence we expect it to contain a tachyon. Another fact that supports this conclusion is that the $U(1) \times U(1)$ gauge fields under which the tachyon is charged do survive the boundary conditions, as we have seen. In a later section we will show explicitly in a T-dual version of this model that the tachyon is indeed present.

Finally we turn to the anti-GSO sector fermions. In the case of infinite $\mathrm{D} 4-\overline{\mathrm{D}} 4$ pairs, we had altogether 32 anti-GSO fermions $\lambda_{A}^{(I)}$, of which 16 were goldstinoes. Now on the suspended D4 - $\overline{\mathrm{D}} 4$ pair we expect to find altogether 16 goldstinoes (because they break all 16 of the supersymmetries that are preserved by the NS5-branes). But so far we have found 8 goldstinoes $\chi_{A}^{+}, A=1,2$ and another 8 non-goldstino fermions $\chi_{A}^{-}=\chi_{A}^{(1)}-\chi_{A}^{(2)}$, $A=1,2$. The remaining 8 goldstinoes must therefore be $\lambda_{A}^{+}, A=1,2$. The symmetry between $\lambda_{A}^{(1)}$ and $\lambda_{A}^{(2)}$ then suggests that the combinations $\lambda_{A}^{-}=\lambda_{A}^{(1)}-\lambda_{A}^{(2)}, A=1,2$ also survive as (non-goldstino) massless fermions.

To summarize, we have a tachyonic $U(1) \times U(1)$ gauge theory on the world-volume of the $\mathrm{D} 4-\overline{\mathrm{D}} 4$ pair, with a pair of gauge fields $A_{\mu}^{(I)}$, massless scalars $X_{i}^{(I)}$ and massless fermions $\chi_{A}^{ \pm}, \lambda_{A}^{ \pm}$of which the + superscripts correspond to goldstinoes. Finally there is a complex tachyon $T=T_{1}+i T_{2}$.

Now we are in a position to ask how this configuration can decay. The first and most elementary process is that the tachyon can go to its minimum. In this case, the brane-antibrane pair annihilates completely. While one of the $U(1)$ gauge symmetries gets Higgsed in the process, the other $U(1)$ gets "confined" by the mechanism discussed in Ref. [9]. At the same time, the value of the potential at the tachyonic minimum, $V\left(T_{0}\right)$, is expected to cancel the tension of the annihilating branes. Thus we end up with a BPS configuration consisting of just a pair of parallel NS5-branes. Note that both the brane tensions and the tachyon potential scale by a common factor of $L_{6}$, the separation between the NS5-branes, hence apart from this overall scale factor, we expect $V\left(T_{0}\right)$ to be independent of the coupling constant of the $3+1$ dimensional field theory. This is consistent with a recent analysis showing that upto such a factor the tachyon potential is universal, independent of the background [10].

A less elementary decay mode would be condensation of a tachyonic vortex[11,12]. For infinite or wrapped $\mathrm{D} p-\overline{\mathrm{D}} p$ pairs, the complex tachyon can condense in a topologically stable vortex, while the relative $U(1)$ gauge field under which it is charged carries a unit of magnetic flux. The result is a stable $\operatorname{BPS} \mathrm{D}(p-2)$ brane. Looking at the field content of the world-volume theory on the suspended $\mathrm{D} 4-\overline{\mathrm{D}} 4$ pair, we see that the same configuration 
is allowed here, providing the resulting D2-brane is extended in the $x^{6}$ direction. The configuration of Fig.(2.1) can therefore decay into a configuration of a BPS D2-brane suspended between parallel NS5-branes.

The picture of this process on the world-volume of the NS5-branes is interesting. A D4-brane ends as a 3-brane in the NS5-brane world-volume. This 3-brane behaves as a vortex since it has co-dimension 2 . The $\overline{\mathrm{D}} 4$ brane similarly behaves as an antivortex. The process where the tachyon goes to its minimum corresponds to simple annihilation of the vortex-antivortex pair. On the other hand, the more complex process in which the tachyon condenses as a vortex, corresponds to the 3-brane anti-3-brane vortices annihilating into a 1-brane1. This 1-brane in the NS5 world-volume is just the end of a D2-brane.

Next let us examine a decay mode which would be allowed for infinite or wrapped D4 $-\overline{\mathrm{D}} 4$ pairs but turns out to be forbidden in the present situation. It has been noted [13] that any $\mathrm{D} p-\overline{\mathrm{D}} p$ pair can also decay by creating an electric flux on its world-volume. In this case, the decay product is a fundamental string (F-string). If this were possible, the configuration of Fig.(2.1) would decay into a configuration where an F-string is suspended between two NS5-branes. Clearly, this is impossible since F-strings cannot end on NS5branes. Hence we must show that such an electric flux is prohibited in the world-volume theory discussed above for Fig.(2.1).

This follows from the fact that the boundary conditions imposed by NS5-branes project out the component $A_{6}^{(I)}$ from each of the two 5-dimensional gauge fields. On D4 - $\overline{\mathrm{D}} 4$ pairs without boundaries, this component would dualize to form part of a magnetic 2-form gauge potential, under which tachyonic strings arising from stretched D2branes are charged (although this phenomenon is, of course, inherently non-perturbative). These magnetic tachyons could then condense, forming an electric world-volume flux [13] (the dual of the usual vortex condensation where an electric tachyon condenses giving rise to a magnetic flux). This process corresponds to the annihilation of the pair into a fundamental string. Once the NS5-branes are put in as boundaries, components of the magnetic 3-form field strength $H_{\mu \nu \rho}$ with $\mu, \nu, \rho \neq 6$ are lost, along with the possibility of an electric flux in the $x^{6}$ direction. However, one is still left with the decay mode in which the $\mathrm{D} 4-\overline{\mathrm{D}} 4$ pairs decay into a fundamental string oriented parallel to the NS5-branes in

1 This discussion may be a little confusing since there are two types of vortices involved. The tachyonic vortex is localized in, say, the $\left(x^{1}, x^{2}\right)$ directions, while the 3 -brane vortices in the NS5-branes are localized in the $\left(x^{4}, x^{5}\right)$ directions. 
either the $x^{1}, x^{2}$ or $x^{3}$ directions. As with similar decays in the case of infinitely extended branes, the resulting configuration preserves some fraction of supersymmetry which, in the present situation, is $1 / 4$ of the original 32 supercharges.

The fact that the D4 - $\overline{\mathrm{D}} 4$ pair cannot decay into a fundamental string stretched between NS5-branes can also be understood in terms of the theory on the NS5-brane worldvolume. In this language we start with a 3-brane vortex-antivortex pair. This cannot annihilate into a point-like object (representing the end of a fundamental string), since a fundamental string carries $B_{N S, N S}$ charge and therefore its endpoint must be an electrically charged particle - but the NS5-brane of type IIA string theory does not carry 1-form gauge fields, and therefore it does not support electrical charges.

For our next example, consider a D3 - $\bar{D} 3$ pair suspended between NS5-branes in type IIB string theory (Fig.(2.2)). The system is very similar to the one above. The NS5-branes have the same locations as before, while the 3 -branes extend along $\left(x^{1}, x^{2}, x^{6}\right)$ and are located at definite positions in $\left(x^{3}, x^{4}, x^{5}\right)$ and at the origin in $\left(x^{7}, x^{8}, x^{9}\right)$. Again, without the antibrane this model would be a familiar one - it belongs to the class of brane constructions analysed in Ref.[14].

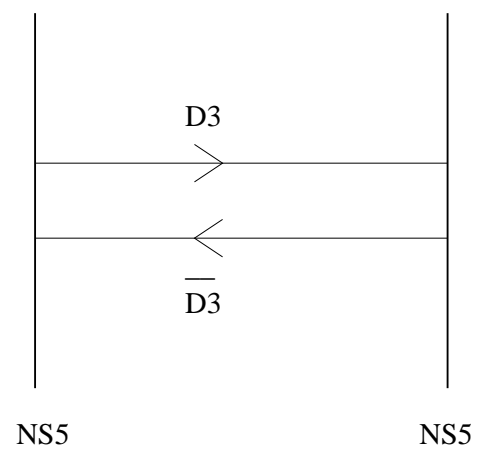

Fig.(2.2): A D3 - $\bar{D} 3$ pair suspended between parallel NS5-branes in type IIB.

The world-volume theory now lives in $2+1$ dimensions. The relevant fields from the GSO-projected sector are a pair of $U(1)$ gauge fields, a set of scalar triplets $X_{i}^{(I)}, i=3,4,5$, and a set of massless fermions $\chi_{A}^{(I)}, A=1, \ldots, 4$. Note that this time the fermions are two-component spinors. From the anti-GSO sector we again expect a complex tachyon $T$ and a set of massless fermions $\lambda_{A}^{(I)}, A=1, \ldots, 4$. In this model, too, the possibilities are that the D3 - $\overline{\mathrm{D}} 3$ pair annihilates completely (with the tachyon going to the minimum of the potential), or there is a tachyonic vortex resulting in annihilation of the D3 - $\overline{\mathrm{D}} 3$ pair into a D-string stretched in the $x^{6}$ direction. As in the model of Fig.(2.1), the loss of $A_{6}$ 
due to boundary conditions ensures the D3 - $\overline{\mathrm{D}} 3$ pair can only decay into an F-string that lies parallel to the NS5-branes.

In this model, it is natural to wonder whether more general decays to (p,q)-strings occur. S-duality ensures that such a decay is allowed in the case of infinitely extended D3 $-\overline{\mathrm{D}} 3$ pairs, with the electric and magnetic tachyons winding $\mathrm{p}$ and $\mathrm{q}$ times respectively before condensing. Notice that the restrictions on the stable decay products discussed above are precisely those of supersymmetry. The corresponding supersymmetry restriction for a (p,q)-string is a configuration in which the string "kinks" between the two NS5branes 15. Unfortunately, we do not have enough understanding of the non-perturbative magnetic tachyon dynamics to say whether such a decay actually occurs.

The third model that we want to consider is quite different. Here we have a D3 - $\overline{\mathrm{D}} 3$ pair suspended between two D5-branes (Fig.(2.3)).

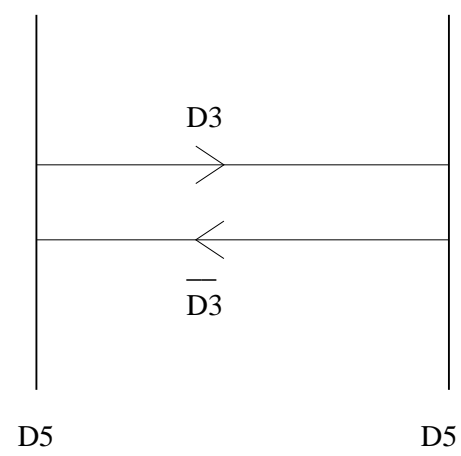

Fig.(2.3): A D3 - $\overline{\mathrm{D}} 3$ pair suspended between parallel D5-branes in type IIB.

In this case, the gauge field component $A_{6}$ is retained while the components $A_{\mu}$, $\mu=0,1,2$ are projected out [14]. The scalar $A_{6}$ may be shifted by an integer multiple of $2 \pi / L_{6}$ by performing a "large" gauge transformation, which acts upon any charged fields by shifting each Kaluza-Klein mode into the next. Thus the scalar $A_{6}$ becomes periodic日 and, as a result, this model has a magnetic rather than electric gauge group. Although the model of Fig.(2.3) is the S-dual of the model of Fig.(2.2), we cannot make any definite use of this fact since we are dealing with unstable non-BPS brane configurations. Sduality relates Fig.(2.2) at weak coupling to Fig.(2.3) at strong coupling and vice-versa, while we are interested in both models at weak coupling. The dynamics of the model of Fig.(2.3) at strong coupling is rather clear: there is a magnetic tachyon, corresponding

2 A well-known example of this fact is that the moduli space of a D-string stretched between D3's is the moduli space of a single monopole, which is $R^{3} \times S^{1}$, with periodicity $2 \pi / L$. 
to quantization of a D-string connecting the D3 - $\overline{\mathrm{D}} 3$ pair, and this can condense with an electric vortex to give an F-string suspended between the D5-branes. This process is S-dual to the condensation of an electric tachyon via a magnetic vortex in the model of Fig.(2.2), and the resulting configuration (which is BPS) is likewise S-dual to a D-string suspended between parallel NS5-branes.

The first statement that we can make about the model of Fig.(2.3) at weak coupling is that the boundary conditions imposed by the D5-branes on the D3 - $\overline{\mathrm{D}} 3$ pair must forbid the decay of this pair into a D-string stretched between the two D5-branes. It is interesting to consider all the available possibilities that lead to this conclusion.

One possibility might be that the complex tachyon is projected out along with the electric gauge field. In this case the D3 - $\bar{D} 3$ pair suspended between two D5-branes would be stable for sufficiently close D5-branes (since it is the constant mode of the tachyon that suffers projection, one could still have a tachyonic instability for well-separated D5-branes). This would mean that suspending a D3 - $\overline{\mathrm{D}} 3$ between D5-branes gives rise to a stable, nonBPS state for some values of the separations. However, the present system is T-dual to a D-string-anti-D-string pair suspended between parallel D3-branes. In that system, the end-point of the D-string behaves as a magnetic monopole in the D3-brane world-volume, so the string-antistring pair is really like a monopole-antimonopole pair. Such a system can certainly annihilate to the vacuum and we therefore expect a tachyon to be present in the spectrum.

A second possibility is that, although the electric gauge field under which the tachyon was charged is projected out by the boundary conditions at the D5-brane, the complex tachyon itself survives. In this scenario, the D3 - $\overline{\mathrm{D}} 3$ pair can annihilate into the vacuum by having the tachyon go to a constant minimum as usual. However, there also exists the possibility of the tachyon winding before condensing, potentially forming the forbidden configuration of a D-string stretched between D5-branes. One might hope that the resulting vortex solution has divergent energy since finite-energy vortices cannot exist in standard field theories without a gauge field. In this case, we would not be able to identify the resulting configuration as a D-string. Moreover, the D-string charge coming from the Chern-Simons coupling $\int F \wedge C_{R R}^{(2)}$ would also be absent without a gauge field.

However, there are some reasons to be dissatisfied with this scenario. Finite-energy vortices without gauge fields are possible if one allows higher-derivative couplings in the field theory, which are certainly present because of stringy corrections. Moreover, besides the Chern-Simons term described above, there is another proposed coupling [16] of the form 
$\int d\left(T D T^{*}\right) \wedge C_{R R}^{(2)}$ on a brane-antibrane pair, which could give rise to the induced D-string charge without the help of a gauge field. Finally, it is believed[1] that a D3 - $\overline{\mathrm{D}} 3$ pair can decay by condensing a real unstable tachyonic kink, into an unstable D2-brane. In turn, this can decay, by condensing a real stable tachyonic kink, into a BPS D-string. This two-step process could again lead to the forbidden D-string, so (barring some mechanism that we do not presently understand that inhibits one of the steps), the hypothesis that the complex tachyon survives seems unlikely.

The final possibility is that one real component of the tachyon is projected out, along with the electric gauge field. The surviving field content of the suspended D3 - $\bar{D} 3$ pair would then be very similar to that of a single unstable D-brane: a U(1) gauge field and scalars, and a neutral real tachyon. This has the advantage that the pair still has two decay modes, out of the original three: it can decay into the vacuum, by condensation of this real tachyon into its constant minimum, or it can decay into the unstable D2-brane suspended between D5-branes by condensation of a real tachyonic kink. Only vortex condensation (whether in one step or two steps) is ruled out since there is only one tachyon.

This possibility seems the most elegant, because it inhibits only the decay mode that is definitely forbidden on grounds of charge conservation, into a D-string. Moreover, it suggests that an unstable D2-brane of type IIB suspended between D5-branes is stable. We will return to this point in the next section.

There is one remaining decay mode to consider in which the D3 - $\overline{\mathrm{D}} 3$ pair decays into a D-string lying parallel to the D5-branes. Such a decay occurs if either a real or complex constant tachyonic mode survives and the topology required to realise these strings as solitonic solutions lies in the periodicity of $A_{6}$. The 3 -dimensional field theory on the D3branes includes the coupling $\left|A_{6}\right|^{2}|T|^{2}$ which ensures that when the tachyon condenses, the vacuum lies at $A_{6}=0$. However, $A_{6}$ may wind as, say, $x_{1}$ ranges from $-\infty$ to $+\infty$, resulting in a string like configuration stretched in the $x_{2}$ direction. This configuration has non-zero flux $F_{16}$ and so, by the usual Chern-Simons term, $\int F \wedge C_{R R}^{(2)}$, is identified as a D-string.

There is another way to see the appearance of strings in this case 3 that dates back to the work of Polyakov [17]. The periodic scalar, $A_{6}$, may be dualised in favour of a 3 -dimensional gauge field, $\widetilde{A}$. The tachyon couples magnetically to $\widetilde{A}$ and, by the dualMeissner effect, condensation of the tachyon leads to a vacuum in which objects charged

3 We thank Kimyeong Lee and Piljin Yi for explanations of this point. 
electrically under $\widetilde{A}$ are linearly confined. The electric flux lines associated with this confinement are then identified with the kinks described above. Similar systems, in which confining flux lines have a description in terms of classical solitons, have been considered recently in the supersymmetric context in [18].

For our final model, consider a D3 - $\overline{\mathrm{D}} 3$ pair suspended between an NS5-brane at one end and a D5-brane at the other. The NS5-brane fills the directions $\left(x^{1}, x^{2}, x^{3}, x^{4}, x^{5}\right)$ and is located at $\left(x^{6}, x^{7}, x^{8}, x^{9}\right)=(0,0,0,0)$, while the D5-brane fills $\left(x^{1}, x^{2}, x^{7}, x^{8}, x^{9}\right)$ and is located at $\left(x^{3}, x^{4}, x^{5}, x^{6}\right)=\left(0,0,0, L_{6}\right)$. The D3 $-\overline{\mathrm{D}} 3$ pair fills the directions $\left(x^{1}, x^{2}\right)$, stretches from $x^{6}=0$ to $x^{6}=L_{6}$ and is located at $\left(x^{3}, x^{4}, x^{5}, x^{7}, x^{8}, x^{9}\right)=(0,0,0,0,0,0)$ (Fig.(2.4)).

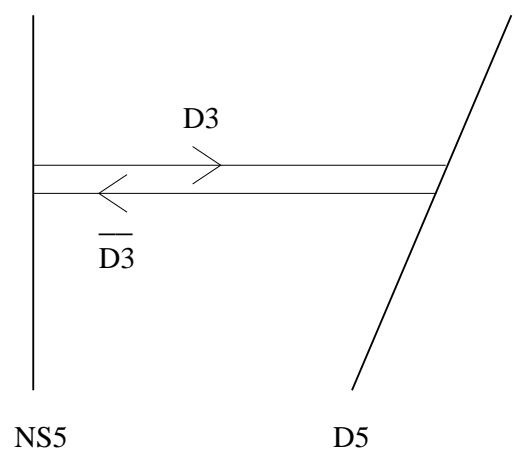

Fig.(2.4): A D3 - $\bar{D} 3$ pair suspended between an NS5 and a D5-brane in type IIB.

From Ref.[14], we know that the massless states in the GSO-projected sector, corresponding to open strings on the brane or antibrane alone, get projected out at one or the other end so there is neither an electric nor a magnetic gauge group. For the anti-GSO sector, we again expect, as above, that either a real tachyon or the whole complex tachyon survives at the D5 end. Whichever of these two possibilities is realised will govern the dynamics of the system, since the whole complex tachyon survives at the NS5-brane end. Note that this model is self-dual under S-duality, so its strong-coupling behaviour will be the same as its weak-coupling behaviour with the roles of the two bounding 5-branes exchanged.

\section{Suspended Unstable D-branes}

The type IIA string has unstable, non-BPS D $p$-branes for $p=1,3,5,7,9$ [1]. Hence one can consider a brane construction in which one of these is suspended between NS5-branes. For example, let us start with an unstable D3-brane suspended in this way (Fig.(3.1)). 


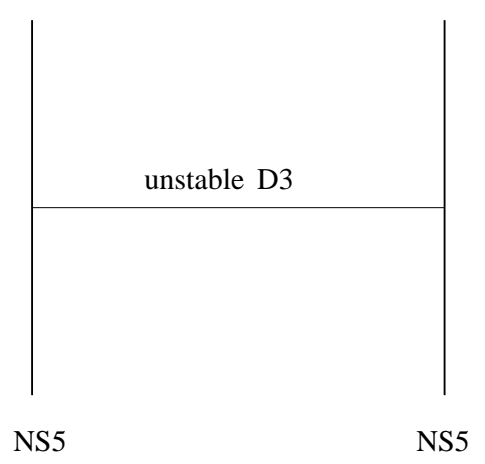

Fig.(3.1): An unstable D3-brane suspended between parallel NS5-branes in type IIA.

The directions of the branes are exactly as for the model in Fig.(2.2). Indeed, the configuration of Fig.(3.1) can be obtained by starting with Fig.(2.2) and taking the quotient of the configuration and the bulk theory together by $(-1)^{F_{L}}$. This is possible since the brane construction in Fig. $(2.2)$ is invariant under $(-1)^{F_{L}}$, which in turn holds since $(-1)^{F_{L}}$ interchanges a D3-brane with a $\overline{\mathrm{D}} 3$-brane in type IIB, and preserves the NS5-brane.

The spectrum on the world-volume theory of Fig.(3.1) is a truncation of that on a single unstable D3-brane of type IIA. This unstable brane, if it is infinite or wrapped, has a single gauge field $A_{\mu}$, a set of massless scalars $X_{i}$ and a set of massless fermions $\chi_{A}$, where $\mu=0, \ldots, 3, i=4, \ldots, 9$ and $A=1, \ldots, 4$. This is a GSO-projected spectrum and arises in the sector with identity Chan-Paton factor. In addition, there is another sector, with Chan-Paton factor $\sigma_{1}$ and anti-GSO projection, where one finds a real tachyon and another set of massless fermions $\lambda_{A}, A=1, \ldots, 4$. This time, all the 32 fermions $\chi_{A}$ and $\lambda_{A}$ are goldstinoes of spontaneously broken supersymmetry. Once the unstable brane is bounded by NS5-branes, it breaks only 16 supersymmetries (the other 16 are already broken by the NS5-branes which act as a "background" from the point of view of the D3-brane). Thus we expect 16 massless fermions $\chi_{A}$ and $\lambda_{A}$ where now $A=1,2$ and all these fermions are goldstinoes. The rest of the light spectrum is made up by the gauge field $A_{\mu}$ and three scalars, $X_{i}, i=3,4,5$, along with a real tachyon $T$.

There is another way to obtain the configuration of Fig.(3.1): start with the configuration of Fig.(1.1), in type IIA theory, and allow the real part of the (complex) tachyon on the D4 - $\overline{\mathrm{D}} 4$ pair to develop a kink. This is not a topologically stable solution, and therefore does not cause the D4- $\overline{\mathrm{D}} 4$ to condense to a stable object. Instead it creates an unstable D3-brane of type IIA at the point where the kink is located[1]. To get the directions appropriate to Fig.(3.1), the kink in Fig.(1.1) must be along the $x^{3}$ direction. 
Since one way of obtaining Fig.(3.1) was to quotient the configuration of Fig.(2.2) by $(-1)^{F_{L}}$, one is tempted to ask whether a similar quotient can be carried out on Figs.(2.1) and (2.3). For Fig.(2.1) this is indeed possible, and one ends up with the unstable D4brane of type IIB suspended between NS5-branes (Fig.(3.2)). However, for Fig.(2.3) the story is quite different. The action of $(-1)^{F_{L}}$ does not preserve D5-branes, hence it is not a symmetry of Fig.(2.3) and one cannot quotient by it.

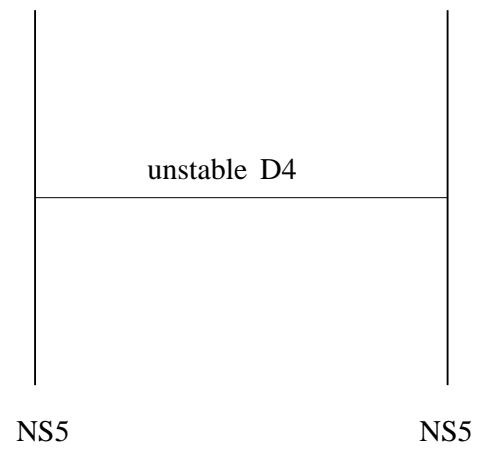

Fig. (3.2): An unstable D4-brane suspended between parallel NS5-branes in type IIB.

Finally one can ask the reverse question: what happens when we quotient Fig.(3.1) by $(-1)^{F_{L}}$ ? It is known that this action takes the unstable D3-brane of type IIA to the stable BPS D3-brane of type IIB. Hence it is reasonable to expect that quotienting Fig.(3.1) by $(-1)^{F_{L}}$ gives us a single BPS D3-brane stretched between NS5-branes in type IIB theory. Likewise, we could take this quotient on Fig.(1.1) and we would end up with a stable D4brane of type IIA suspended between two NS5-branes. Thus, the stable BPS configurations analysed in Refs. 8, 14 arise as $(-1)^{F_{L}}$ quotients of the unstable configurations considered in this section.

Since unstable branes do not carry charges, one may ask if they can always be consistently suspended between any pair of stable branes. Consider a D3 - $\overline{\mathrm{D}} 3$ pair suspended between NS5-branes in type IIB. Clearly, condensation of a real tachyonic kink can give rise to an unstable D2-brane suspended between the NS5-branes. The tachyon that condenses is the real part of an electric tachyon on the D3- D 3 pair, which as we have argued, survives the boundary conditions imposed by the NS5-brane. Thus it must be consistent to have an unstable D2-brane suspended between NS5-branes, analogous to the system in Fig.(3.2). (This configuration can also be produced by starting with a D2 - $\overline{\mathrm{D}} 2$ pair in type IIA, suspended between NS5-branes, and quotienting by $(-1)^{F_{L}}$.) Moreover, the tachyon on this D2-brane, although uncharged, is actually the imaginary part of the electric tachyon on the original D3 - $\overline{\mathrm{D}} 3$ pair. Thus, this tachyon is also not projected out 
by the NS5-brane, and it can undergo further kink condensation leading to a stable BPS configuration, a D-string suspended between the NS5-branes.

If we replace the NS5-branes by D5-branes, we certainly cannot produce such a configuration by starting from type IIA and quotienting by $(-1)^{F_{L}}$, since type IIA has no BPS D5-branes. Thus the only way to produce this configuration will be by kink condensation starting from a D3 - $\overline{\mathrm{D}} 3$ pair stretched between the D5-branes. This leads to two possibilities, which are linked to the two possibilities considered in the previous section. If the D3 - $\overline{\mathrm{D}} 3$ pair retains a complex tachyon, then condensation of a real kink will give rise to the unstable D2-brane suspended between D5-branes. However, as noted in the previous section, this does give rise to a potential paradox. The D2-brane would in turn be tachyonic, and its decay could potentially produce a BPS D-string suspended between D5-branes, which we know to be inconsistent.

A more plausible scenario, in the light of our discussion in the previous section, arises if the D3 - $\overline{\mathrm{D}} 3$ pair between D5-branes retains only one real tachyon. In that case, condensation of a tachyonic kink would lead to a D2-brane suspended between D5-branes, which in turn would be stable because there is no second tachyon. An amusing feature

of this scenario is that instead of the usual 3-step chain: D3 - $\overline{\mathrm{D}} 3 \rightarrow \mathrm{D} 2 \rightarrow \mathrm{D} 1$, where the successive elements have two tachyons, one tachyon and no tachyon, we find a shorter chain: suspended D3 - D $3 \rightarrow$ suspended D2, where the two elements have respectively one tachyon and no tachyon.

In this situation, note that the suspended D2-brane not only has no 2-brane charge (which is the defining property of an unstable D-brane) but also no induced lower-form charges. Such charges can only arise via world-volume Chern-Simons couplings involving a tachyon and various even-form RR potentials, so once the tachyon is projected out, this leaves a totally uncharged object.

\section{Suspended Adjacent Brane-Antibrane Pairs}

In this section we introduce three parallel NS5-branes in type IIA theory and consider a system in which a D4-brane and a $\overline{\mathrm{D}} 4$-brane end on a common NS5-brane from opposite sides. The configuration is that of Fig.(4.1). 


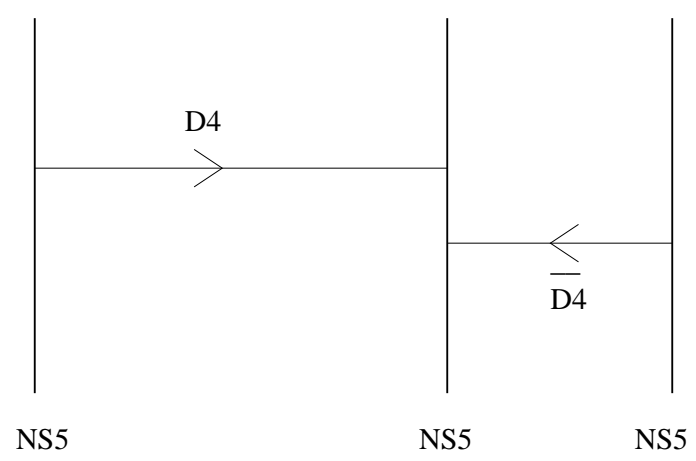

Fig.(4.1): Adjacent D4 - $\overline{\mathrm{D}} 4$ pairs in type IIA.

This type of configuration is much harder to analyse. Even for the BPS analogue (with a D-brane on either side of an NS5-brane), it is difficult to reliably extract the spectrum, and one has to use the fact that one can continuously interpolate to a different configuration of intersecting branes where perturbation theory is reliable 14. Alternatively one can use duality, as we will do in subsequent sections.

The question we want to address is whether this configuration has an instability, and if so, what is its nature and to what final configuration does the system tend. On physical grounds, we might expect the system to be tachyonic and unstable. If there is an attractive force between the brane-antibrane pair, then they will tend to line up as in Fig.(4.2).

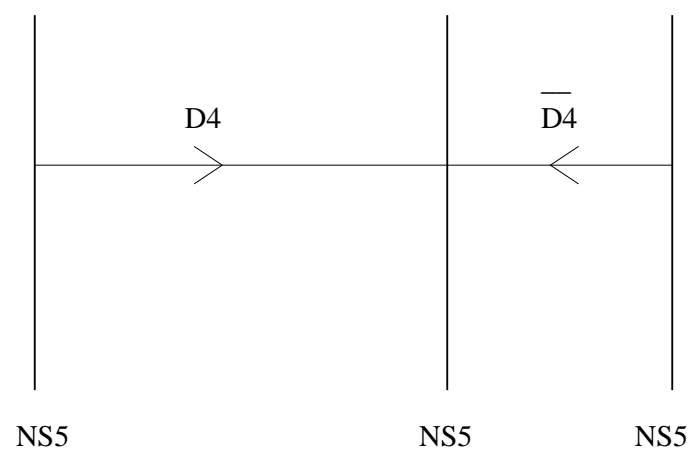

Fig.(4.2): Lining up of adjacent D4 - $\overline{\mathrm{D}} 4$ pairs in type IIA.

On the other hand, if the force is repulsive then they will move apart. From the point of view of one of the pair, the other will go to infinity.

Rather than immediately studying the above problem, we turn first to a related system that is more amenable to calculation: that of an adjacent $\mathrm{D} 1-\overline{\mathrm{D}} 1$ pair suspended between D3-branes. This configuration is related to the above by S- and T-dualities. However, the need to perform an S-duality, relating strong and weak coupling regimes, means that the 
physics of this system is not necessarily the same as that of Fig.(4.1). Nevertheless, in a later section we will return to the configuration of Fig.(4.1) and find the same behaviour.

The D3-branes support an $\mathcal{N}=4, d=3+1$-dimensional $U(3)$ gauge theory. The D-string is a magnetic monopole of charges $(1,-1,0)$ under the $U(1) \times U(1) \times U(1)$ Cartan subalgebra of $U(3)$, while the $\overline{\mathrm{D}}$-string is a monopole of charges $(0,-1,1)$. In terms of the $S U(3)$ subgroup of $U(3)$ (neglecting the centre-of-mass factor), the configuration is like a monopole of one $U(1)$ and an anti-monopole of the other. With this description, one may calculate the force between the two D-strings by treating the monopoles as point particles [19]. In fact, the result may be seen quite simply, as both D-string and $\overline{\mathrm{D}}$-string appear as positive charges on the middle D3-brane. We therefore expect them to repel, and this is indeed the case.

Let us now be both more quantitative and more general. Consider an arbitrary simple gauge group $\mathcal{G}$, with a single adjoint Higgs field $\phi$, which acquires a vacuum expectation value $(\mathrm{VEV}),\langle\phi\rangle=\mathbf{h} \cdot \mathbf{H}$, where $\mathbf{H}$ is the rank $r$ dimensional Cartan basis and $\mathbf{h}$ is an $r$-dimensional vector. We assume the $\mathrm{VEV}$ is such that $\mathcal{G}$ is broken to the maximal torus, $U(1)^{r}$.

The vector $\mathbf{h}$ lies in the root space and therefore determines a fundamental Weyl chamber from which we define the simple roots, $\alpha_{j}, j=1, \cdots, r$. Recall that these are the roots that satisfy $\mathbf{h} \cdot \alpha_{j}>0$ and have the property that any other root is a linear combination of the $\alpha$ 's with either all positive or all negative coefficients.

Magnetic monopoles are configurations with magnetic field given asymptotically by,

$$
B_{i}=\mathbf{g} \cdot \mathbf{H} \frac{r_{i}}{4 \pi r^{3}}
$$

The magnetic charge vector $\mathbf{g}$ is forced by topological considerations to lie in the co-root lattice, $\mathbf{g}=4 \pi \sum_{j} n_{j} \alpha_{j}^{\star} / e$, where $n_{j}$ are integers and $e$ is the gauge coupling constant. Whether a given topological charge sector is to be considered as a monopole or antimonopole depends on the sign of $\mathbf{h} \cdot \mathbf{g}$, as can be seen in the most general form of the Bogomol'nyi equation,

$$
B_{i}=\operatorname{sign}(\mathbf{h} \cdot \mathbf{g}) D_{i} \phi
$$

4 Of course, the $U(N)$ gauge theory on D3-brane has 6 adjoint Higgs fields. We assume that all branes are co-linear. The force between two D-strings (as opposed to D-string and anti-D-string) when this is not the case has been calculated in [20]. 
In particular, all charges equal to linear combinations of positive co-roots are monopoles, while those equal to linear combinations of negative co-roots are anti-monopoles. We will be interested in charges that are given by the sum of a positive co-root and negative coroot. Notice that for $r>1$, there are non-trivial topological sectors with this property. This contrasts with the case of $S U(2)$ gauge group where a monopole-antimonopole pair necessarily lies in the sector with zero topological charge.

The method used to calculate the long-range force between two static monopoles of magnetic charges $\mathbf{g}_{1}$ and $\mathbf{g}_{2}$ separated by a distance $r$, is well known [19]. Treating the monopoles as point particles, they interact through two massless fields: the gauge field and the Higgs field. We treat each in turn. Firstly, the magneto-static potential is given by

$$
V_{e m}=\mathbf{g}_{1} \cdot \mathbf{g}_{2} \frac{1}{4 \pi r}
$$

Secondly, the potential due to the massless scalar field is determined by approximating the configuration of two monopoles by a simple superposition of the individual solutions. Using Eqn. (4.1) and (4.2), the asymptotic form of the Higgs field of the second monopole is given by,

$$
\phi=\mathbf{h} \cdot \mathbf{H}-\operatorname{sign}(\mathbf{h} \cdot \mathbf{g}) \frac{1}{4 \pi r} \mathbf{g} \cdot \mathbf{H}
$$

The potential is calculated by examining the energy of the first monopole which, when isolated, is given by $M=\left\|\mathbf{h} \cdot \mathbf{g}_{1}\right\|$. However, in the presence of the second monopole, the effective mass of the first becomes,

$$
M_{1}^{e f f}=\left\|\left(\mathbf{h}-\operatorname{sign}\left(\mathbf{h} \cdot \mathbf{g}_{2}\right) \frac{1}{4 \pi r} \mathbf{g}_{2}\right) \cdot \mathbf{g}_{\mathbf{1}}\right\|
$$

which, for large separation $r \gg 1 /\|\mathbf{h}\|$, has an expansion as

$$
\begin{aligned}
M_{1}^{e f f} & =\left\|\mathbf{h} \cdot \mathbf{g}_{1}\right\|-\operatorname{sign}\left(\mathbf{h} \cdot \mathbf{g}_{1}\right) \operatorname{sign}\left(\mathbf{h} \cdot \mathbf{g}_{2}\right) \frac{\mathbf{g}_{1} \cdot \mathbf{g}_{2}}{4 \pi r} \\
& =M_{1}+V_{\text {Higgs }}
\end{aligned}
$$

The full potential between two well separated monopoles of charges $\mathbf{g}_{1}$ and $\mathbf{g}_{2}$ is therefore

$$
V=V_{e m}+V_{H i g g s}=\frac{\mathbf{g}_{1} \cdot \mathbf{g}_{2}}{4 \pi r}\left(1-\operatorname{sign}\left(\mathbf{h} \cdot \mathbf{g}_{1}\right) \operatorname{sign}\left(\mathbf{h} \cdot \mathbf{g}_{2}\right)\right)
$$

Let us now illustrate this formula with a few simple examples. Firstly, consider an $S U(2)$ gauge theory with the VEV $h>0$. We have two possibilities: $g_{1}=g_{2}=1$ or $g_{1}=-g_{2}=1$. 
In the first situation, the potential vanishes. This is simply the well-known cancellation of the force between two BPS monopoles. In the second situation however, the magneto-static and Higgs potentials combine to produce a negative potential, reflecting the attractive force between a monopole-antimonopole pair. This is the relevant situation for Fig.(2.3).

Now applying Eqn.(4.7) to the case at hand, we choose $\mathbf{h}$ such that the simple roots of $S U(3)$ are $(1,-1,0)$ and $(0,1,-1)$. The sector we are interested in consists of a monopole of charge $\mathbf{g}_{1}=(1,-1,0)$, and another of charge $\mathbf{g}_{2}=(0,-1,1)$. We therefore have $\operatorname{sign}\left(\mathbf{h} \cdot \mathbf{g}_{1}\right)=+1$ while $\operatorname{sign}\left(\mathbf{h} \cdot \mathbf{g}_{2}\right)=-1$ and again we see non-cancellation between the magneto-static and Higgs forces. However, now the potential is positive. The adjacent D-string and anti-D-string therefore repel.

This result is interesting in itself, but we can ask what it tells us about the original system of an adjacent D $4-\overline{\mathrm{D}} 4$ pair. As we observed already, these calculations do not apply directly to the system that we started out with, because the configuration they describe is related to our original one only after S-duality. However, we can make two relevant observations. One is that if we take a system of parallel D5-branes in type IIB and suspend a D3 brane in one interval and a $\overline{\mathrm{D}} 3$ in the next, then we only need Tdualities, and no S-dualities, to relate that system to the $S U(3)$ monopole-antimonopole pair studied above. In this way we can remain at weak coupling throughout, hence the above computation reliably tells us that an adjacent brane-antibrane pair separated by a D5-brane does repel.

The second observation is that even for an adjacent brane-antibrane pair separated by an NS5-brane, the qualitative reason for repulsion still holds: charges of the same sign are deposited on the middle brane, while on the outer branes the product of deposited charges is zero. Thus, just by analysing charges, one sees that the pair should repel. We will confirm this by dualising to fractional branes in a subsequent section.

An interesting extension of the above model(s) arises as follows. Supposing we start with four parallel NS5-branes in type IIA theory. Then we can suspend a D4-brane between the first two and a $\overline{\mathrm{D}} 4$-brane between the next two (Fig.(4.3)). In this situation the D4- $\overline{\mathrm{D}} 4$ pair is not adjacent, but separated by an "empty" interval. The arguments of the previous section, based on charges, suggest that at tree level there is neither attraction nor repulsion between the D4- $\overline{\mathrm{D}} 4$ pair in this model. Hence we appear to have found a stable, non-BPS configuration from brane-antibrane constructions. Indeed, in the related D1-D3 system, there exists a corresponding non-BPS solution of the classical field equations, consisting of a monopole and anti-monopole placed in commuting $S U(2)$ subgroups. 


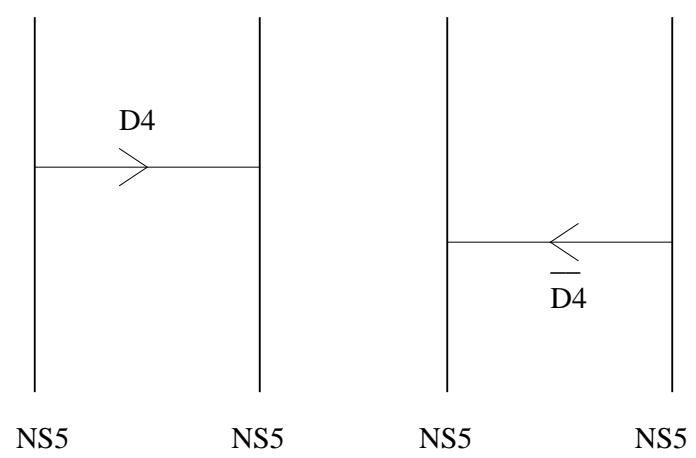

Fig.(4.3): Non-adjacent D4 - $\overline{\mathrm{D}} 4$ pairs in type IIA.

Note that in this system, the locations of the first two NS5-branes in $x^{7}, x^{8}, x^{9}$ must be equal to the location of the D4-brane in these directions, while the locations of the next two NS5-branes in these directions must likewise be equal to that of the $\overline{\mathrm{D}} 4$-brane ending on them. However, these two sets of locations need not be equal to each other.

This system can be shown to be T-dual to a $Z_{4}$ ALE singularity. The above D4 and $\overline{\mathrm{D}} 4$ branes correspond to two of the eight different fractional branes that arise when a full D4 - $\overline{\mathrm{D}} 4$ pair is brought to a $Z_{4}$ singularity. The spectrum of light states for strings stretching between such a pair can in principle be analysed using quiver techniques, though we will not do so in this paper.

Note that if we replace the D4 and $\overline{\mathrm{D}} 4$ branes with D2 and $\overline{\mathrm{D}} 2$ branes respectively, we end up with a system that is similar to one that was investigated in Ref. [21]. In the latter, a well-separated pair of $Z_{2}$ ALE singularities arranged at opposite ends of a circle is shown to support a stable non-BPS state, consisting of a fractional D2-brane wrapped on the first singularity and a $\overline{\mathrm{D}} 2$-brane wrapped on the second. As the singularities get closer, this system is unstable to decay into a single D-string of type IIA wrapped on the circle that connects them. As we will see, well-separated ALE singularities can be T-dualised to a set of NS5-branes on a very small circle, which is precisely the system being considered in this paper. The limit of a small circle ensures that the approximation of a common world-volume field theory is valid. While the system described in Fig.(4.3) is not dual to a pair of $Z_{2}$ singularites, for some purposes a $Z_{4}$ singularity with the two end cycles shrunk to zero size should provide similar physics. It would be interesting to explore whether a similar phase diagram to the one studied in Ref. [22] arises in the $Z_{4}$ model. 


\section{Borromean Branes}

Consider now a configuration in type IIB string theory containing NS5-branes, D5branes and D3-branes all together. The branes are aligned as follows:

$$
\begin{aligned}
\text { NS5 : } & \left(x^{1}, x^{2}, x^{3}, x^{4}, x^{5}\right) \\
\text { D5 : } & \left(x^{1}, x^{2}, x^{7}, x^{8}, x^{9}\right) \\
\text { D3 : } & \left(x^{1}, x^{2}, x^{6}\right)
\end{aligned}
$$

This is the class of configurations studied by Hanany and Witten[14], and following them we also use the notation $\vec{x}=\left(x^{3}, x^{4}, x^{5}\right)$ and $\vec{y}=\left(x^{7}, x^{8}, x^{9}\right)$. Scalar fields representing translational modes in these directions will be denoted $\vec{X}$ and $\vec{Y}$.

To start with, consider two parallel NS5-branes at the same value of $\vec{y}$, say $\vec{y}=\overrightarrow{0}$, and separated by a finite distance in $x^{6}$. Suspend a D3-brane between them. This can be located at any value of $\vec{x}$, though it must have $\vec{y}=0$ to end on the NS5-branes. At some intermediate value of $x^{6}$, insert a D5-brane at a fixed $\vec{x}$, say $\vec{x}=\overrightarrow{0}$. The whole configuration is shown in Fig.(5.1).

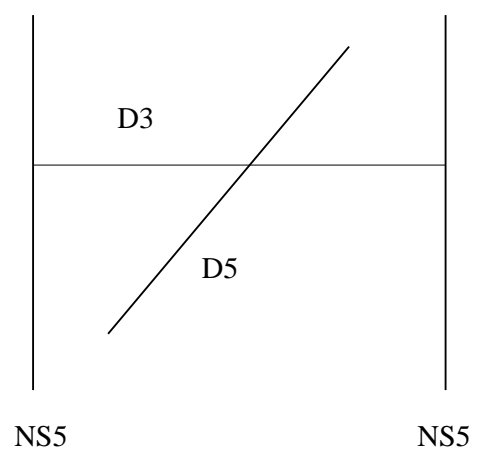

Fig.(5.1): Hanany-Witten configuration.

Any two of the three branes in this construction break the supersymmetry to $\frac{1}{4}$ of the maximal value, so the field theory on the common intersection will have 8 supersymmetries. Adding the third brane does not further reduce the number of supersymmetries. To illustrate this, split the 32 supercharges of IIB string theory into four groups of 8 , labelled $Q_{\lambda}, \lambda=1, \cdots, 4$. Each brane breaks half of the supersymmetry as indicated in the following table,

$\begin{array}{ccccc} & Q_{1} & Q_{2} & Q_{3} & Q_{4} \\ N S 5 & \times & \sqrt{ } & \times & \sqrt{ } \\ D 5 & \sqrt{ } & \times & \times & \sqrt{ } \\ D 3 & \times & \times & \sqrt{ } & \sqrt{ }\end{array}$


where $\mathrm{a} \times$ denotes that the supersymmetry is broken by a given brane, while a $\sqrt{ }$ means that it is preserved. We see that the 8 supercharges of $Q_{4}$ are preserved by all branes, and we therefore have a $2+1$-dimensional field theory with $\mathcal{N}=4$ supersymmetry describing the low energy dynamics of the D3-brane.

The massless spectrum of this $2+1$-dimensional field theory is as follows 14. Firstly, the 3-brane suspended between two parallel NS5-branes has a pure $\mathcal{N}=4$ supersymmetric $U(1)$ gauge theory, containing a gauge field, three scalars associated to the transverse motion of the 3 -brane in the $\vec{x}$ directions, and fermions. Inserting the D5-brane in the middle gives rise to a charged hypermultiplet from the open string connecting it to the D3-brane. This hypermultiplet becomes massless when the D3-brane moves to $\vec{x}=0$, where it touches the D5-brane.

Now let us ask what happens if we replace the D3-brane with a $\bar{D} 3$-brane, while keeping the NS5 and D5 branes unchanged (Fig.(5.2) 5 . The supersymmetries preserved by the system may be read off from Table (5.2), simply by exchanging each $\times$ and $\sqrt{ }$ in the D3-brane row, to get

$\begin{array}{ccccc} & Q_{1} & Q_{2} & Q_{3} & Q_{4} \\ N S 5 & \times & \sqrt{ } & \times & \sqrt{ } \\ D 5 & \sqrt{ } & \times & \times & \sqrt{ } \\ \overline{\mathrm{D}} 3 & \sqrt{ } & \sqrt{ } & \times & \times\end{array}$

The system now breaks all supersymmetries. Notice however that any pair of branes still preserves 8 supercharges. We call this configuration "Borromean" (in analogy with the famous topological configuration of three rings, where any two are unlinked but all three together are linked). We may restore supersymmetry by changing either of the 5-branes to an antibrane. If however we change both D5 and NS5-branes to $\overline{\mathrm{D}} 5$ and $\overline{\mathrm{NS}} 5$-branes respectively, so that all branes in Fig.(5.1) have been changed to antibranes, we once again have a situation preserving no supersymmetry.

\footnotetext{
5 This configuration was described in Ref. 223.
} 


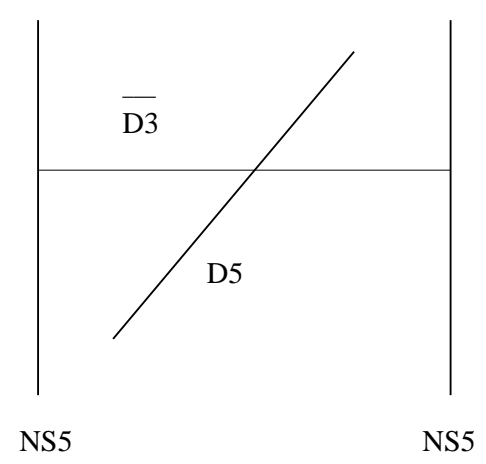

Fig.(5.2): Borromean branes.

Let us now analyse the spectrum of the Borromean brane system. Since the $\overline{\mathrm{D}} 3$-brane suspended between two NS5-branes is supersymmetric, it will give rise to an $\mathcal{N}=4 U(1)$ vector multiplet. On the other hand, if we consider the $\overline{\mathrm{D}} 3$-brane along with the D5-brane, the pair is also supersymmetric and the open string joining them gives rise, as previously, to an $\mathcal{N}=4$ hypermultiplet. Since the light fields all come from considering two of the three branes at a time, we see that there is no tachyon in the one-particle Hilbert space of this theory. However, although pairwise supersymmetric, the coupling of the hypermultiplet to the vector multiplet does not preserve supersymmetry. We will now argue that the correct non-supersymmetric $d=2+1$-dimensional low-energy gauge theory is given by the usual $\mathcal{N}=4$ Lagrangian without the Yukawa terms coupling a hypermultiplet scalar and fermion to a vector multiplet fermion.

To see this we use the pairwise supersymmetry of the configuration to determine which fermions on the worldvolume of the D3-brane couple to the 3-5 strings, and which are projected out by the boundary conditions of the NS5-branes. Let us begin with the latter problem and consider an infinite 3-brane. The world-volume fields on this brane are all goldstone modes for broken symmetries: 6 scalars, $\vec{X}$ and $\vec{Y}$, and 16 goldstinoes (corresponding to the supercharges $Q_{1}$ and $Q_{2}$ for the D3-brane of Eqn.(5.2), and to $Q_{3}$ and $Q_{4}$ for the $\overline{\mathrm{D}} 3$-brane of Eqn.(5.3)). The presence of the NS5-branes on which the 3 -brane ends projects out half of these fields. Of the bosons, the surviving fields are $\vec{X}$, which are the space-time directions which do not arise as goldstone bosons on the NS5brane, together with the $A_{0}, A_{1}$ and $A_{2}$ component of the gauge field. By supersymmetry, the surviving fermions are those goldstinoes which arise from supersymmetries broken by the 3-brane, but preserved by the NS5-branes. In the case of the D3-brane, this means $Q_{2}$, while for the $\overline{\mathrm{D}} 3$-brane, it is $Q_{4}$. 
Now let us examine the hypermultiplet fields arising from the $3-5$ string. We are interested in determining to which fields on the 3-brane they couple. Once again, we consider first the case of an infinite 3-brane. The world-volume theory is $d=3+1$, $\mathcal{N}=4 U(1)$ gauge theory. The presence of the D5-brane breaks this supersymmetry by half and the $\mathcal{N}=4$ multiplet splits into an $\mathcal{N}=2$ vector multiplet and a neutral $\mathcal{N}=2$ hypermultiplet. The manner in which this split occurs is determined by the fact that the charged hypermultiplet arising from the 3-5 string is minimally coupled only to the former. Notice that as the D5-brane is not Lorentz invariant from the point of view of an infinite 3-brane world-volume, the gauge field will also be split, partly living in the vector multiplet and partly in the hypermultiplet. More specifically, the 3-5 string couples to the components of the gauge field $A_{0}, A_{1}$ and $A_{2}$, together with the scalars $\vec{X}$. To see the latter is particularly simple, as motion of the 3-brane in the $\vec{x}$ directions stretches the $3-5$ string and so gives a mass to the charged hypermultiplet. Stated differently, the charged 3-5 hypermultiplet couples to those scalars which are goldstone modes for both the 3-brane and the D5-brane. By supersymmetry, it therefore also couples to the fermions on the 3-brane which are goldstinoes for both 3-brane and D5-brane. Hence, in the case of the D3-brane in (5.2), the 3-5 string couples to $Q_{2}$, while for the $\overline{\mathrm{D}} 3$-brane of $(5.3)$, it couples to $Q_{3}$.

Putting these two facts together allows us to determine the field theory for the Borromean branes of Fig.(5.2). The 3-5 string couples to the scalars $\vec{X}$, the gauge fields $A_{0}, A_{1}, A_{2}$ and the goldstinoes $Q_{3}$. However, the NS5-branes project out all fields except for the above bosons and the goldstinoes $Q_{4}$. In particular, the fermions to which the hypermultiplet couples are lost due to the boundary conditions imposed by the NS5-brane. Therefore, the gauge theory on the D3-brane is as in the supersymmetric case, except that the Yukawa terms coupling the 3-5 string hypermultiplet to the 3-3 string fermions is missing, thus breaking supersymmetry.

Having identified the non-supersymmetric gauge theory living on the D3-brane, we must now determine the correct vacuum state of this theory. Classically, the theory has flat directions given by the vacuum expectation values of the scalars $\vec{X}$ and the dual photon. As the theory is non-supersymmetric, the flat directions associated to the scalars $\vec{X}$ are not protected against quantum corrections. However, they do remain at one-loop as a consequence of bose-fermi degeneracy. Whether this cancellation continues at higher loops is an open question. 
One may pass from the Borromean brane situation to that of adjacent brane-antibrane pairs, generalising those discussed in the previous section, by passing the D5-brane through one of the NS5-branes. The pairwise supersymmetry of these two 5-branes ensures that the Hanany-Witten transition[14] occurs as in the supersymmetric case, and we are left with a D3-brane suspended between the D5 and NS5-brane (Fig.(5.3)).

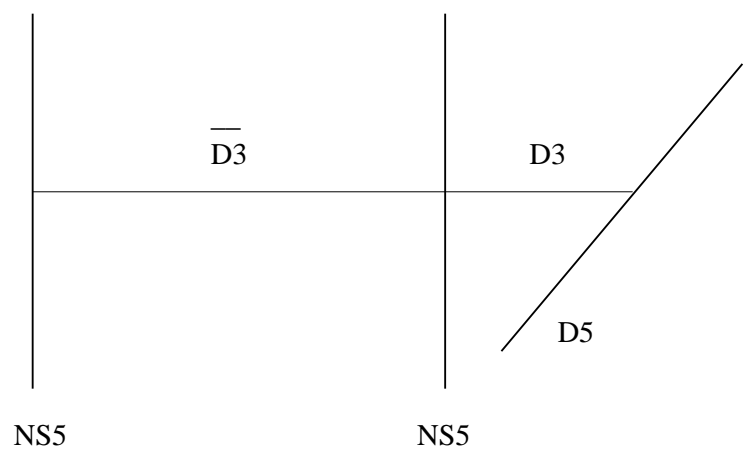

Fig.(5.3): Result of Hanany-Witten transition on Borromean branes.

The brane configuration is no longer Borromean, as the D3 and $\overline{\mathrm{D}} 3$-branes are not pairwise supersymmetric. Applying the considerations of the previous section locally to the region near the middle 5-brane, we would expect that the D3 - $\overline{\mathrm{D}} 3$ pair repels. However, it is quite possible that in this model the Hanany-Witten transition is accompanied by a phase transition, so we are unable to relate the physics of Figs.(5.2) and (5.3). Note in particular that the dimensionless expansion parameter in the theory on the Borromean branes is $e^{2} / r$, where $e$ is the gauge coupling constant, and $r$ the distance between the D5-brane and $\overline{\mathrm{D}} 3$-brane. The one-loop flatness of the vacuum moduli space of this theory ensures that any potential that is generated at large distance is of the form $\pm 1 / r^{n}$, for $n \geq 2$. This is to be contrasted with the $1 / r$ behaviour of adjacent brane-antibrane pairs separated by a D5-brane.

\section{Dualising NS5-branes into ALE spaces}

Consider type IIA string theory compactified on a 4-torus $T^{4}$ in the directions $x^{6}, x^{7}, x^{8}, x^{9}$ and orbifolded by the inversion $I_{6789}$. This gives rise to 16 fixed orbifold 5-planes. Each of these is locally a $Z_{2}$ ALE space. Or goal is to dualise this system and then, by going to a suitable region of moduli space, to isolate the dual of a single $Z_{2}$ ALE space. The material in this section is not new, having been discussed in slightly different language in Refs.[6] and in the context of conifold singularities in Refs. [24,25]. The duality 
that we discuss below will be used in the subsequent section to map the suspended brane systems to brane-antibrane generalisations of quiver theories.

Under T-duality along $x^{6}$, the inversion $I_{6789}$ is mapped to $(-1)^{F_{L}} I_{6789}$, as one can easily see by examining the action on various massless states. At the same time, the type IIA theory is mapped to the type IIB theory. Thus the dual to the original orbifold is the orbifold of type IIB string theory on a 4 -torus quotiented by $(-1)^{F_{L}} I_{6789}$. This too has 16 fixed points, but the nature of the orbifold 5-planes is quite different. To see this, perform an S-duality. The operator $(-1)^{F_{L}}$ is mapped to the orientifolding operation $\Omega$, and the resulting theory has 16 orientifold 5-planes (O5-planes) along with 16 (mirror pairs of) D5-branes. The D5-branes can sit on the orientifold planes to make charge-cancelled configurations, or they can move off. Hence it must be that, before performing S-duality, the 16 orbifold planes were made up of NS5-branes (which are S-dual to D5-branes in type IIB) and static planes that are S-dual to O5-planes. We call these "S5-planes".

To summarise, the T-dual of type IIA on a $T^{4} / Z_{2}$ orbifold is type IIB on a 4 -torus with 16 S5-planes and 16 pairs of NS5-branes. At the orbifold point of the type IIA theory, the flux of the 2 -form $B$-field through each $Z_{2}$ singularity is $\frac{1}{2}$, while on the IIB side, each NS5-brane is on top of an S5-plane. Now since the T-duality was performed only on $x^{6}$, we can identify the $x^{7}, x^{8}, x^{9}$ positions on the two sides and decompactify them. As a result we have on the type IIA side, a pair of $Z_{2}$ ALE spaces located symmetrically around the $x^{6}$ circle, and at the origin of $x^{7}, x^{8}, x^{9}$, while on the type IIB side there is a pair of S5-planes similarly located symmetrically around the dual $x^{6}$ circle, and a pair of NS5-branes at points of this circle (Fig.(6.1)).
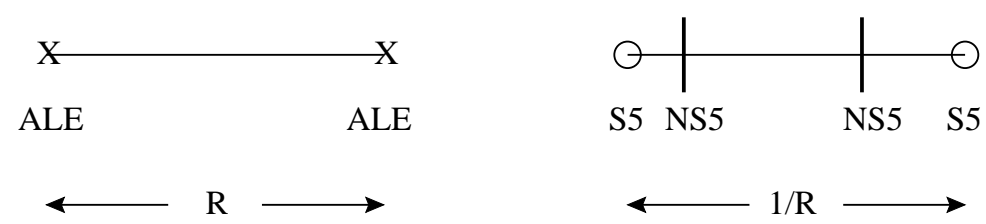

Fig.(6.1): Duality between $Z_{2}$ ALE spaces and S5-NS5 systems.

The matching of moduli is reasonably straightforward. In particular, if $R$ is the radius of the $x^{6}$ circle on the type IIA side then the dual radius is $\frac{1}{R}$. Let $B, B^{\prime}$ be the fluxes of 
the $B$-field through the two $Z_{2}$ ALE spaces on the IIA side, and let $X_{6}^{(1)}, X_{6}^{(2)}$ be the $x_{6}$ locations of the two NS5-branes on the IIB side. Then we have:

$$
\begin{aligned}
& X_{6}^{(1)}=\frac{1}{R}\left|B-B^{\prime}\right| \\
& X_{6}^{(2)}=\frac{1}{R}\left|B+B^{\prime}\right|
\end{aligned}
$$

Note that with this matching, the type IIA theory has an enhanced gauge symmetry if $B=0$ or $B^{\prime}=0$, coming from a BPS D2-brane wrapping the 2-cycle of the first or second ALE space, and this corresponds in type IIB to $X_{6}^{(1)}=X_{6}^{(2)}$ so the two NS5-branes meet. If both $B=B^{\prime}=0$ then we have $S U(2) \times S U(2)$ enhanced gauge symmetry, and on the type IIB side, $X_{6}^{(1)}=X_{6}^{(2)}=0$, so the two NS5-branes meet on an S5-plane, where in fact we expect $S O(4) \sim S U(2) \times S U(2)$.

Suppose we now fix $B^{\prime}=\frac{1}{2}$ once and for all. Then no enhanced gauge symmetry will ever arise from the second ALE space, and henceforth we focus our attention on the region near the first ALE space. The dual picture now has the two NS5-branes at equal distances from their corresponding S5-planes. They can only meet far away from the S5-planes (in fact, at the midpoint of the strip depicted in Fig.(6.1)). Hence the S5 orbifold planes can be ignored. The result is a duality between a pair of NS5-branes arranged around a circle (in type IIB) and a solitary $Z_{2}$ ALE singularity (in type IIA). The roles of type IIA and type IIB can also be reversed.

Now we may wrap a $\mathrm{D} p$-brane around the $x^{6}$ circle and perform the above duality. It turns into a $\mathrm{D}(p-1)$ brane at the $Z_{2}$ ALE singularity. Breaking the original $p$-brane on the NS5-branes corresponds to breaking the $(p-1)$-brane into a pair of fractional branes, which are free to move around while remaining in the 5-plane of the ALE singularity.

In a similar fashion, one can start with a 2-torus slanted at a particular angle and carry out a $Z_{k}$ quotient $z \rightarrow \omega z$ where $\omega^{k}=1$. Near the origin, the system can be described in T-dual language by a set of $k$ NS5-branes located around the $x^{6}$ circle. The separations of the NS5-branes along $x^{6}$ are given by B-fields through the various 2-cycles of the $Z_{k}$ singularity, while their locations in $x^{7}, x^{8}, x^{9}$ are determined by the geometrical size parameters of these 2-cycles. 


\section{Brane-Antibrane Pairs at an ALE Singularity}

In this section we obtain the spectrum of light fields (including possible tachyons) on brane-antibrane pairs placed at ALE quotient singularites. For simplicity we will work mainly with the case of $R^{4} / Z_{2}$. We start with this singularity, where $R^{4}$ corresponds to the directions $x^{6}, x^{7}, x^{8}, x^{9}$, and bring a $\mathrm{D} p-\overline{\mathrm{D}} p$ pair close to it ( $p$ is even in type IIA and odd in type IIB). Our analysis will closely parallel that of Ref. [7] where BPS D-branes at ALE spaces were studied. Some aspects of brane-antibrane systems at ALE spaces have been investigated previously in Ref. [26].

In order to work out the spectrum of the resulting theory, we first introduce the "mirror" of the $\mathrm{D} p-\overline{\mathrm{D}} p$ pair in the $Z_{2}$ singularity, thus making four branes altogether. Let these four branes be labelled $1,1^{\prime}, \overline{1}, \overline{1}^{\prime}$, where the prime denotes a mirror brane and the bar denotes an antibrane. Thus there are $4 \times 4$ Chan-Paton factors, which we classify as follows. We use the $2 \times 2$ Chan-Paton labels $A, B, C, D$ to distinguish between strings on a brane, on a mirror brane, or between the two, while the labels $a, b, c, d$ are used (as before) to distinguish between strings on a brane, on an antibrane, or between the two. The correspondence between $a, b, c, d$ and Chan-Paton matrices was given in Eqn.(2.1), and the analogous correspondence holds also for $A, B, C, D$.

With these definitions, we have the correspondence:

$$
\begin{array}{cccc}
1-1: A \otimes a & \overline{1}-\overline{1}: A \otimes b & 1-\overline{1}: A \otimes c & \overline{1}-1: A \otimes d \\
1-1^{\prime}: C \otimes a & \overline{1}-\overline{1}^{\prime}: C \otimes b & 1-\overline{1}^{\prime}: C \otimes c & \overline{1}-1^{\prime}: C \otimes d \\
1^{\prime}-1: D \otimes a & \overline{1}^{\prime}-\overline{1}^{\prime}: D \otimes b & 1^{\prime}-\overline{1}: D \otimes c & \overline{1}^{\prime}-1: D \otimes d \\
1^{\prime}-1^{\prime}: B \otimes a & \overline{1}^{\prime}-\overline{1}^{\prime}: B \otimes b & 1^{\prime}-\overline{1}^{\prime}: B \otimes c & \overline{1}^{\prime}-1^{\prime}: B \otimes d
\end{array}
$$

The spectrum of this system, before projection by the $Z_{2}$, contains gauge fields $A_{\mu}^{(I)}$, $\mu=0, \ldots, p$, along with scalars $X_{i}^{(I)}, i=p+1, \ldots, 5$, and $X_{m}^{(I)}, m=6,7,8,9$. These fields all lie in the adjoint of $U(2) \times U(2)$, with the superscript $(I)$ labelling the relevant factor. Along with the obvious fermionic counterparts, these make up a set of massless fields arising in the Chan-Paton sectors that appear in the first two columns of Eqn.(7.1). Each Chan-Paton sector in the remaining two columns gives rise to a tachyon $T$, hence there are 8 real tachyons altogether. These are accompanied by a set of massless Ramond fermions which are oppositely GSO-projected as compared to the ones that accompanied the gauge field and massless scalars. 
There are two interesting $Z_{2}$ involutions: the inversion that creates the ALE space, which we denote by $I_{6789}$, and the symmetry $(-1)^{F_{L}}$ which we have used before. In our conventions, these involutions act as conjugation by:

$$
\begin{aligned}
I_{6789} & : \sigma_{1} \otimes 1 \\
(-1)^{F_{L}} & : 1 \otimes \sigma_{1}
\end{aligned}
$$

This follows from the fact that the first $\sigma_{1}$ factor exchanges a brane with its mirror, while the second $\sigma_{1}$ exhanges a brane with an antibrane.

It is now straightforward to write down the transformations of the various fields under $I_{6789}$ :

$\begin{array}{lll}\underline{\text { Field }} & \underline{\text { CP factor }} & \underline{I_{6789}} \\ A_{\mu}^{(I)}, X_{i}^{(I)} & \left\{1, \sigma_{1}\right\} \otimes\left\{1, \sigma_{3}\right\} & + \\ X_{m}^{(I)} & \left\{i \sigma_{2}, \sigma_{3}\right\} \otimes\left\{1, \sigma_{3}\right\} & + \\ A_{\mu}^{(I)}, X_{i}^{(I)} & \left\{i \sigma_{2}, \sigma_{3}\right\} \otimes\left\{1, \sigma_{3}\right\} & - \\ X_{m}^{(I)} & \left\{1, \sigma_{1}\right\} \otimes\left\{1, \sigma_{3}\right\} & - \\ T & \left\{1, \sigma_{1}\right\} \otimes\left\{\sigma_{1}, i \sigma_{2}\right\} & + \\ T & \left\{i \sigma_{2}, \sigma_{3}\right\} \otimes\left\{\sigma_{1}, i \sigma_{2}\right\} & -\end{array}$

Thus under orbifolding by $I_{6789}$, the fields which are invariant survive, while the others are projected out. The gauge group that survives (for a brane-antibrane pair on top of the orbifold singularity) is $U(1)^{4}$. This statement really applies when all vev's are zero, which is the common origin of the Higgs branch and Coulomb branch. We see from the table that 4 real tachyons survive in the orbifolded theory, these can be grouped into two complex tachyons that transform as the bi-fundamental of the first $U(1) \times U(1)$ and the second $U(1) \times U(1)$ respectively.

On the Higgs branch, the brane-antibrane pair leaves the orbifold plane and the gauge group is Higgsed to $U(1) \times U(1)$. This corresponds to retaining the following fields from the above table:

$$
\begin{aligned}
A_{\mu}^{(I)}, X_{i}^{(I)}: & 1 \otimes\left\{1, \sigma_{3}\right\} \\
X_{m}^{(I)}: & \sigma_{3} \otimes\left\{1, \sigma_{3}\right\} \\
T: & 1 \otimes\left\{\sigma_{1}, i \sigma_{2}\right\}
\end{aligned}
$$

(For $X_{m}$ this involves a gauge choice, see Ref.[27].) Thus on this branch there is a single complex tachyon, transforming as the bi-fundamental of $U(1) \times U(1)$. 
We can relate all this to the brane constructions that we discussed above. Compactify $x^{6}$ on a circle and T-dualize, then we have a pair of NS5-branes on the circle and a $\mathrm{D}(p+1)-\overline{\mathrm{D}}(p+1)$ pair running all the way around the circle (Fig.(6.1)).

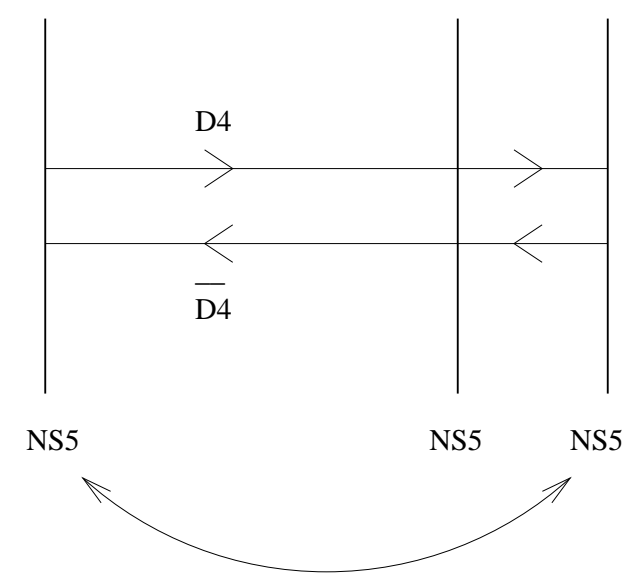

Fig.(7.1): A pair of NS5-branes and a $\mathrm{D}(p+1)-\overline{\mathrm{D}}(p+1)$ pair wrapped on $x^{6}$.

The figure describes the point where the Higgs branch and Coulomb branch meet, and it is clear that the gauge group should be $U(1)^{4}$ as we have shown. Moreover, in this brane construction one expects 2 complex tachyons, corresponding to open strings connecting a brane and antibrane segment suspended between the same NS5-branes. They manifestly carry the right charges. Moving onto the Higgs branch is accomplished by taking the braneantibrane pair away from the NS5-branes, this breaks the gauge group to $U(1) \times U(1)$ and there is just one complex tachyon.

To understand the Coulomb branch, observe that both the brane and the antibrane which wrap all the way around $x^{6}$ in the dual brane construction can break on the NS5branes. (In the T-dual picture this corresponds to the fact that branes at an ALE singularity can break into fractional branes [27, [2,28,29]. Hence, altogether we have four brane segments in the problem (or rather, two brane segments and two antibrane segments). We can separate all these and take some of the segments off to infinity. For example, suppose we take a brane and an antibrane stretching along the same segment to infinity. The result (after taking the radius very large) will be the diagram of Fig.(2.1). On the other hand, we can take away an antibrane from one segment and a brane from the adjacent segment. The result will be an adjacent brane-antibrane pair, similar to the construction in Fig.(4.1). We will use these facts in the T-dual picture to understand more about the brane constructions of Figs.(2.1),(4.1). 
Returning to the $\mathrm{D} p-\overline{\mathrm{D}} p$ pair at an ALE singularity, we should now identify the ChanPaton factors corresponding to strings connecting fractional branes. Recall that for a single $\mathrm{D} p$-brane at a $Z_{2}$ singularity, the Coulomb branch arises when $X_{m}=0$ and $X_{i} \sim\left\{1, \sigma_{1}\right\}$. It is on this branch that the single $\mathrm{D} p$-brane is said to split into a pair of fractional branes and the gauge group becomes $U(1) \times U(1)$. Note that the individual gauge fields associated to the two fractional branes are associated to the Chan-Paton factors $1+\sigma_{1}$ and $1-\sigma_{1}$ respectively.

For a $\mathrm{D} p-\overline{\mathrm{D}} p$ system, the Higgs branch is described by $X_{m}^{(I)} \neq 0, X_{i}^{(I)} \sim 1 \otimes\left\{1, \sigma_{3}\right\}$ while the Coulomb branch is $X_{m}^{(I)}=0, X_{i}^{(I)} \sim\left\{1, \sigma_{1}\right\} \otimes\left\{1, \sigma_{3}\right\}$. This time, as expected, the latter branch describes four fractional branes altogether. Let us denote these fractional branes as $1_{f}, 1_{f}^{\prime}, \overline{1}_{f}$ and $\overline{1}_{f}^{\prime}$ (note that in this case, the prime does not denote the mirror image, though the bar still denotes the antibrane). The interpretations of these branes are as follows: $1_{f}$ is a $(\mathrm{p}+2)$-brane wrapped on the vanishing 2 -cycle $\Sigma$ at the ALE singularity 6 . $1_{f}^{\prime}$ is an anti- $(\mathrm{p}+2)$-brane wrapped on the same 2 -cycle. On the other hand, $\overline{1}_{f}$ is an anti$(\mathrm{p}+2)$-brane, while $\overline{1}_{f}^{\prime}$ is a $(\mathrm{p}+2)$-brane.

Following the discussion in Ref. [29], we can assign the following world-volume couplings to the four fractional branes:

$$
\begin{array}{ll}
1_{f}: & \int\left(C_{R R}^{(p+3)}+\left(B_{N S}-F_{1_{f}}\right)\right) \wedge C_{R R}^{(p+1)} \\
1_{f}^{\prime}: & \int\left(-C_{R R}^{(p+3)}-\left(B_{N S}-F_{1_{f}^{\prime}}\right)\right) \wedge C_{R R}^{(p+1)} \\
\overline{1}_{f}: & \int\left(-C_{R R}^{(p+3)}-\left(B_{N S}-F_{\overline{1}_{f}}\right)\right) \wedge C_{R R}^{(p+1)} \\
\overline{1}_{f}^{\prime}: & \int\left(C_{R R}^{(p+3)}+\left(B_{N S}-F_{\overline{1}_{f}^{\prime}}\right)\right) \wedge C_{R R}^{(p+1)}
\end{array}
$$

Moreover, the world-volume field strengths are given by $\int_{\Sigma} F_{1_{f}}=\int_{\Sigma} F_{\overline{1}_{f}}=0$ and $\int_{\Sigma} F_{1_{f}^{\prime}}=$ $\int_{\Sigma} F_{\overline{1}_{f}^{\prime}}=1$.

We would like to find the spectrum arising from all possible configurations of open strings on this set of four fractional branes. This can be read off directly from (7.3). First

6 At the orbifold point, we have an unresolved orbifold singularity through which the $B$-field has a flux of $\frac{1}{2}$. This also means that the NS5-branes are symmetrically placed along the $x^{6}$ circle in the T-dual construction. However, we can of course allow $B$ to vary, and in the following discussion we have in mind this more general case. 
consider open strings starting and ending on the same brane. These each carry a gauge field $A_{\mu}$ and a pair of scalars $X_{i}(i=4,5)$ having Chan-Paton factors:

$$
\begin{aligned}
1_{f}-1_{f}: & \frac{1}{2}\left(1+\sigma_{1}\right) \otimes \frac{1}{2}\left(1+\sigma_{3}\right) \\
1_{f}^{\prime}-1_{f}^{\prime}: & \frac{1}{2}\left(1-\sigma_{1}\right) \otimes \frac{1}{2}\left(1+\sigma_{3}\right) \\
\overline{1}_{f}-\overline{1}_{f}: & \frac{1}{2}\left(1+\sigma_{1}\right) \otimes \frac{1}{2}\left(1-\sigma_{3}\right) \\
\overline{1}_{f}^{\prime}-\overline{1}_{f}^{\prime}: & \frac{1}{2}\left(1-\sigma_{1}\right) \otimes \frac{1}{2}\left(1-\sigma_{3}\right)
\end{aligned}
$$

Next, we have in principle 6 oriented strings going from each one of the four fractional branes to another, and each string has two orientations, for a total of 12 . However, some of these are projected out. In fact, we get massless hypermultiplet scalars $X_{m}$ as follows:

$$
\begin{aligned}
1_{f}-1_{f}^{\prime}: & \frac{1}{2}\left(\sigma_{3}+i \sigma_{2}\right) \otimes \frac{1}{2}\left(1+\sigma_{3}\right) \\
1_{f}^{\prime}-1_{f}: & \frac{1}{2}\left(\sigma_{3}-i \sigma_{2}\right) \otimes \frac{1}{2}\left(1+\sigma_{3}\right) \\
\overline{1}_{f}-\overline{1}_{f}^{\prime}: & \frac{1}{2}\left(\sigma_{3}+i \sigma_{2}\right) \otimes \frac{1}{2}\left(1-\sigma_{3}\right) \\
\overline{1}_{f}^{\prime}-\overline{1}_{f}: & \frac{1}{2}\left(\sigma_{3}-i \sigma_{2}\right) \otimes \frac{1}{2}\left(1-\sigma_{3}\right)
\end{aligned}
$$

Tachyons arise from the following open strings:

$$
\begin{aligned}
1_{f}-\overline{1}_{f}: & \frac{1}{2}\left(1+\sigma_{1}\right) \otimes \frac{1}{2}\left(\sigma_{1}+i \sigma_{2}\right) \\
\overline{1}_{f}-1_{f}: & \frac{1}{2}\left(1+\sigma_{1}\right) \otimes \frac{1}{2}\left(\sigma_{1}-i \sigma_{2}\right) \\
1_{f}^{\prime}-\overline{1}_{f}^{\prime}: & \frac{1}{2}\left(1-\sigma_{1}\right) \otimes \frac{1}{2}\left(\sigma_{1}+i \sigma_{2}\right) \\
\overline{1}_{f}^{\prime}-1_{f}^{\prime}: & \frac{1}{2}\left(1-\sigma_{1}\right) \otimes \frac{1}{2}\left(\sigma_{1}-i \sigma_{2}\right)
\end{aligned}
$$

Thus there are four real tachyons in the Coulomb branch. The remaining open strings 
correspond in principle to the following Chan-Paton factors:

$$
\begin{aligned}
1_{f}-\overline{1}_{f}^{\prime}: & \frac{1}{2}\left(\sigma_{3}+i \sigma_{2}\right) \otimes\left(\sigma_{1}+i \sigma_{2}\right) \\
\overline{1}_{f}^{\prime}-1_{f}: & \frac{1}{2}\left(\sigma_{3}-i \sigma_{2}\right) \otimes\left(\sigma_{1}-i \sigma_{2}\right) \\
1_{f}^{\prime}-\overline{1}_{f}: & \frac{1}{2}\left(\sigma_{3}-i \sigma_{2}\right) \otimes\left(\sigma_{1}+i \sigma_{2}\right) \\
\overline{1}_{f}-1_{f}^{\prime}: & \frac{1}{2}\left(\sigma_{3}+i \sigma_{2}\right) \otimes\left(\sigma_{1}-i \sigma_{2}\right)
\end{aligned}
$$

However, as one can see from (7.3), these strings are projected out: the tachyon is removed by $I_{6789}$ and the massless states are removed by the anti-GSO projection. Hence we find the novel result that strings connecting $1_{f}$ to $\overline{1}_{f}^{\prime}$ have no tachyonic or massless bosonic states.

Now let us consider first the system of a suspended $\mathrm{D}(p+1)-\overline{\mathrm{D}}(p+1)$ pair as in Fig.(2.1). This is described in the T-dual version by keeping the fractional branes $1_{f}$ and $\overline{1}_{f}$. We see that for all values of $\int_{\Sigma} B_{N S}$, the total $(\mathrm{p}+2)$-brane and p-brane charges add up to zero. The analysis above tells us that the spectrum on this pair consists of a $U(1) \times U(1)$ gauge field, massless scalars $X_{i}$ and a complex tachyon $T$. All this is consistent with the fact that we expect the original pair to be able to annihilate completely. Thus we confirm the heuristic picture of this system developed in Section 2.

Turning next to the system of an adjacent $\mathrm{D}(p+1)-\overline{\mathrm{D}}(p+1)$ pair as in Fig.(4.1), we see that in this case the dual description is obtained by keeping the fractional branes $1_{f}$ and $\overline{1}_{f}^{\prime}$ (or equivalently $\overline{1}_{f}$ and $1_{f}^{\prime}$, with some sign changes in the following). In this case the picture is very different. The system has a net $(p+2)$-brane charge of +2 , and a net p-brane charge of $2 B_{N S}-1$. The latter vanishes only at the symmetric point $B_{N S}=\frac{1}{2}$, for which the NS5-branes in the original construction are equally spaced around the circle. As far as the spectrum is concerned, there are neither any tachyons nor any massless scalars coming from open strings between the pair.

Finally, one can consider non-adjacent brane-antibrane pairs from this point of view by taking a brane-antibrane pair to a $Z_{4}$ ALE singularity, where it can split into a total of 8 fractional branes. We will not describe this explicitly here. 


\section{Boundary State Computation of Brane-Antibrane Forces}

In this section our aim is to construct the boundary states corresponding to the fractional D3-branes and use them to compute forces between pairs of fractional branes of the various types discussed in the previous section. We will use the conventions of Ref. [30].

For this we have to construct consistent boundary states which describe D3-branes in the $Z_{2}$ orbifold of type IIB string theory where the orbifolding group $Z_{2}$ is generated by $I_{6789}$ (which we refer to as $R$ ). Boundary states at orbifolds have been classified and constructed in great generality in Ref. 31]. Here we will only describe the formulae relevant for our purpose.

Let us consider a D3-brane in type IIB string theory on $R^{9,1}$. Take the D3-brane to be along the $x^{3}, x^{4}, x^{5}$ directions, and situated at the origin in $x^{6}, x^{7}, x^{8}, x^{9}$ directions. We make a double Wick rotation $x^{0} \rightarrow i x^{0}, x^{1} \rightarrow i x^{1}$, so that the light-cone directions are $x^{1} \pm x^{2}$ and the D3-brane world-volume directions are all space-like. We first define the basic boundary states in terms of which the D3-brane state will be defined.

In the untwisted sector these states are:

$$
\begin{aligned}
|k, \eta\rangle_{\substack{N S N S ; U \\
R R ; U}} & =\exp \left(\sum_{n=1}^{\infty}\left[-\frac{1}{n} \sum_{\mu=0,3,4,5} \alpha_{-n}^{\mu} \widetilde{\alpha}_{-n}^{\mu}+\frac{1}{n} \sum_{\mu=6}^{9} \alpha_{-n}^{\mu} \widetilde{\alpha}_{-n}^{\mu}\right]\right. \\
& \left.+i \eta \sum_{r>0}\left[-\sum_{\mu=0,3,4,5} \psi_{-r}^{\mu} \widetilde{\psi}_{-r}^{\mu}+\sum_{\mu=6}^{9} \psi_{-r}^{\mu} \widetilde{\psi}_{-r}^{\mu}\right]\right)|k, \eta\rangle_{\substack{N S N S ; U \\
R R ; U}}^{(0)}
\end{aligned}
$$

where $k \equiv\left(k^{1}, k^{2}, k^{6}, k^{7}, k^{8}, k^{9}\right)$ and $\eta= \pm$. In the NSNS sector, the labels $n, r$ are

$$
\begin{aligned}
& n \in Z_{+} \quad \text { for } \quad \mu=0,3,4, \ldots, 9 \\
& r \in Z_{+}-\frac{1}{2} \quad \text { for } \quad \mu=0,3,4, \ldots, 9
\end{aligned}
$$

while in the RR sector they are:

$$
n, r \in Z_{+} \quad \text { for } \quad \mu=0,3,4, \ldots, 9
$$

The NSNS vacuum state is independent of $\eta$, while the RR vacuum states are defined as:

$$
\begin{aligned}
\psi_{-}^{\mu}|k, \eta\rangle_{R R ; U}^{(0)} & =0 \quad \text { for } \quad \mu=0,3,4,5 . \\
\psi_{+}^{\mu}|k, \eta\rangle_{R R ; U}^{(0)} & =0 \quad \text { for } \quad \mu=6,7,8,9 . \\
|k,+\rangle_{R R ; U}^{(0)} & =\prod_{\mu=6}^{9} \psi_{-}^{\mu} \prod_{\mu=0,3}^{5} \psi_{+}^{\mu}|k,-\rangle_{R R ; U}^{(0)}
\end{aligned}
$$


Next let us turn to the twisted sector of the $Z_{2}$ orbifold. Here the relevant boundary states are given by:

$$
\begin{aligned}
& |k, \eta\rangle_{\substack{N S N S, T, T \\
R R ;, T}}=\exp \left(\sum_{n>0}^{\infty}\left[-\frac{1}{n} \sum_{\mu=0,3,4,5} \alpha_{-n}^{\mu} \widetilde{\alpha}_{-n}^{\mu}+\frac{1}{n} \sum_{\mu=6}^{9} \alpha_{-n}^{\mu} \widetilde{\alpha}_{-n}^{\mu}\right]\right. \\
& \left.+i \eta \sum_{r>0}\left[-\sum_{\mu=0,3,4,5} \psi_{-r}^{\mu} \widetilde{\psi}_{-r}^{\mu}+\sum_{\mu=6}^{9} \psi_{-r}^{\mu} \widetilde{\psi}_{-r}^{\mu}\right]\right)|k, \eta\rangle_{\substack{N S N S ; T \\
R R ; T}}^{(0)}
\end{aligned}
$$

where $k \equiv\left(k^{1}, k^{2}\right)$. In the twisted NSNS sector, the labels $n, r$ are

$$
\begin{aligned}
n & \in Z_{+} & \text {for } & \mu=0,3,4,5, \\
& \in Z_{+}-\frac{1}{2} & \text { for } & \mu=6,7,8,9, \\
r & \in Z_{+}-\frac{1}{2} & \text { for } & \mu=0,3,4,5, \\
& \in Z_{+} & \text {for } & \mu=6,7,8,9 .
\end{aligned}
$$

while in the twisted RR sector they are:

$$
\begin{aligned}
n, r & \in Z_{+} \quad \text { for } \quad \mu=0,3,4,5, \\
& \in Z_{+}-\frac{1}{2} \quad \text { for } \quad \mu=6,7,8,9 .
\end{aligned}
$$

Since in the twisted sector both NSNS and RR sectors have zero modes we have to define the vacua carefully (as we did for the RR sector of the untwisted sector). Define

$$
\begin{aligned}
\psi_{+}^{\mu}|k,-\rangle_{N S N S ; T}^{(0)} & =0 \quad \text { for } \quad \mu=6,7,8,9 . \\
|k,+\rangle_{N S N S ; T}^{(0)} & =\prod_{\mu=6}^{9} \psi_{-}^{\mu}|k,-\rangle_{N S N S ; T}^{(0)}
\end{aligned}
$$

Similarly for the RR vacuum state, define

$$
\begin{gathered}
\psi_{-}^{\mu}|k,-\rangle_{R R ; T}^{(0)}=0 \quad \text { for } \quad \mu=0,3,4,5, \\
|k,+\rangle_{R R ; T}^{(0)}=\prod_{\mu=0,3}^{5} \psi_{+}^{\mu}|k,-\rangle_{R R ; T}^{(0)}
\end{gathered}
$$

Now we integrate these states over momentum, to define states corresponding to 
branes at fixed positions. In the untwisted sector we get:

$$
\begin{aligned}
|\eta\rangle_{N S N S ; U} & =\mathcal{N} \int \prod_{\mu=1,2,6}^{9} d k^{\mu}|k, \eta\rangle_{N S N S ; U} \\
|\eta\rangle_{R R ; U} & =4 i \mathcal{N} \int \prod_{\mu=1,2,6}^{9} d k^{\mu}|k, \eta\rangle_{R R ; U}
\end{aligned}
$$

while the corresponding states in the twisted sector are:

$$
\begin{aligned}
|\eta\rangle_{N S N S ; T} & =2 \widetilde{\mathcal{N}} \int \prod_{\mu=1}^{2} d k^{\mu}|k, \eta\rangle_{N S N S ; T} \\
|\eta\rangle_{R R} & =2 i \widetilde{\mathcal{N}} \int \prod_{\mu=1}^{2} d k^{\mu}|k, \eta\rangle_{R R ; T}
\end{aligned}
$$

Next we combine the above states into the appropriate GSO-invariant linear combinations to describe D3 and $\bar{D} 3$ branes at the ALE space. In the untwisted sector this gives the states:

$$
\begin{aligned}
|U\rangle_{N S N S} & =\frac{1}{\sqrt{2}}\left(|+\rangle_{N S N S ; U}-|-\rangle_{N S N S ; U}\right), \\
|U\rangle_{R R} & =\frac{1}{\sqrt{2}}\left(|+\rangle_{R R ; U}+|-\rangle_{R R ; U}\right),
\end{aligned}
$$

while in the twisted sector the GSO-invariant combinations are:

$$
\begin{aligned}
|T\rangle_{N S N S} & =\frac{1}{\sqrt{2}}\left(|+\rangle_{N S N S ; T}+|-\rangle_{N S N S ; T}\right) \\
|T\rangle_{R R} & =\frac{1}{\sqrt{2}}\left(|+\rangle_{R R ; T}+|-\rangle_{R R ; T}\right)
\end{aligned}
$$

Finally we can combine the untwisted and twisted sector boundary states to produce states that describe branes in the full theory. We find four independent consistent boundary states for D3, $\overline{\mathrm{D}} 3$, which can be identified with the four fractional branes $1_{f}, 1_{f}^{\prime}, \overline{1}_{f}, \overline{1}_{f}^{\prime}$. The states along with their identifications are as follows:

$$
\begin{aligned}
|\mathrm{D} 3,+\rangle=\frac{1}{2}\left(|U\rangle_{N S N S}+|U\rangle_{R R}+|T\rangle_{N S N S}+|T\rangle_{R R}\right) & : 1_{f} \\
|\mathrm{D} 3,-\rangle=\frac{1}{2}\left(|U\rangle_{N S N S}+|U\rangle_{R R}-|T\rangle_{N S N S}-|T\rangle_{R R}\right) & : 1_{f}^{\prime} \\
|\overline{\mathrm{D}} 3,+\rangle=\frac{1}{2}\left(|U\rangle_{N S N S}-|U\rangle_{R R}-|T\rangle_{N S N S}+|T\rangle_{R R}\right) & : \overline{1}_{f}^{\prime} \\
|\overline{\mathrm{D}} 3,-\rangle=\frac{1}{2}\left(|U\rangle_{N S N S}-|U\rangle_{R R}+|T\rangle_{N S N S}-|T\rangle_{R R}\right) & : \overline{1}_{f}
\end{aligned}
$$


Now we can explicitly write down the closed string tree amplitudes between the above boundary states in terms of open string one loop amplitudes. First, we choose $32 \mathcal{N}^{2}=$ $\frac{v^{(4)}}{(2 \pi)^{4}}$, where $v^{(4)}$ is the infinite volume of the brane along the $x^{6}, x^{7}, x^{8}, x^{9}$ directions. This normalization is determined by the requirement that amplitudes between boundary states can be interpreted as open-string traces. Next, define $\widetilde{q}=\exp (-\pi t)$ and

$$
\begin{aligned}
& f_{1}(q)=q^{\frac{1}{12}} \prod_{n=1}^{\infty}\left(1-q^{2 n}\right)=\eta\left(q^{2}\right) \\
& f_{2}(q)=\sqrt{2} q^{\frac{1}{12}} \prod_{n=1}^{\infty}\left(1+q^{2 n}\right)=\sqrt{\frac{\theta_{2}\left(q^{2}\right)}{\eta\left(q^{2}\right)}} \\
& f_{3}(q)=q^{-\frac{1}{24}} \prod_{n=1}^{\infty}\left(1+q^{2 n+1}\right)=\sqrt{\frac{\theta_{3}\left(q^{2}\right)}{\eta\left(q^{2}\right)}} \\
& f_{4}(q)=q^{-\frac{1}{24}} \prod_{n=1}^{\infty}\left(1-q^{2 n+1}\right)=\sqrt{\frac{\theta_{4}\left(q^{2}\right)}{\eta\left(q^{2}\right)}}
\end{aligned}
$$

Then we find:

$$
\begin{aligned}
\int_{0}^{\infty} d l\left\langle\mathrm{D} 3,+\left|e^{-l H_{c}}\right| \mathrm{D} 3,+\right\rangle= & \int_{0}^{\infty} \frac{d t}{2 t} \operatorname{tr}_{N S-R}\left(\frac{1+(-1)^{F}}{2} \frac{1+R}{2} e^{-2 t H_{0}}\right) \\
= & \frac{v^{(4)}}{32(2 \pi)^{4}} \int_{0}^{\infty} \frac{d t}{t^{3}}\left\{\frac{f_{3}(\widetilde{q})^{8}-f_{4}(\widetilde{q})^{8}-f_{2}(\widetilde{q})^{8}}{f_{1}(\widetilde{q})^{8}}\right. \\
& \left.+4 \frac{f_{4}(\widetilde{q})^{4} f_{3}(\widetilde{q})^{4}-f_{4}(\widetilde{q})^{4} f_{3}(\widetilde{q})^{4}}{f_{1}(\widetilde{q})^{4} f_{2}(\widetilde{q})^{4}}\right\}
\end{aligned}
$$

The amplitude between any other fractional brane and itself is given by the same expression. Using the abstruse identity $f_{3}(\widetilde{q})^{8}-f_{4}(\widetilde{q})^{8}-f_{2}(\widetilde{q})^{8}=0$, we see that this amplitude vanishes, as expected.

Next, consider the amplitude:

$$
\begin{aligned}
\int_{0}^{\infty} d l\left\langle\mathrm{D} 3,+\left|e^{-l H_{c}}\right| \mathrm{D} 3,-\right\rangle= & \int_{0}^{\infty} \frac{d t}{2 t} \operatorname{tr}_{N S-R}\left(\frac{1+(-1)^{F}}{2} \frac{1-R}{2} e^{-2 t H_{0}}\right) \\
= & \frac{v^{(4)}}{32(2 \pi)^{4}} \int_{0}^{\infty} \frac{d t}{t^{3}}\left\{\frac{f_{3}(\widetilde{q})^{8}-f_{4}(\widetilde{q})^{8}-f_{2}(\widetilde{q})^{8}}{f_{1}(\widetilde{q})^{8}}\right. \\
& \left.-4 \frac{f_{4}(\widetilde{q})^{4} f_{3}(\widetilde{q})^{4}-f_{4}(\widetilde{q})^{4} f_{3}(\widetilde{q})^{4}}{f_{1}(\widetilde{q})^{4} f_{2}(\widetilde{q})^{4}}\right\}
\end{aligned}
$$


This gives the force between $1_{f}$ and $1_{f}^{\prime}$, and is also equal to zero.

The next amplitude of interest is:

$$
\begin{aligned}
\int_{0}^{\infty} d l\left\langle\mathrm{D} 3,+\left|e^{-l H_{c}}\right| \overline{\mathrm{D}} 3,-\right\rangle= & \int_{0}^{\infty} \frac{d t}{2 t} \operatorname{tr}_{N S-R}\left(\frac{1-(-1)^{F}}{2} \frac{1+R}{2} e^{-2 t H_{0}}\right) \\
= & \frac{v^{(4)}}{32(2 \pi)^{4}} \int_{0}^{\infty} \frac{d t}{t^{3}}\left\{\frac{f_{3}(\widetilde{q})^{8}+f_{4}(\widetilde{q})^{8}-f_{2}(\widetilde{q})^{8}}{f_{1}(\widetilde{q})^{8}}\right. \\
& \left.+4 \frac{f_{4}(\widetilde{q})^{4} f_{3}(\widetilde{q})^{4}+f_{4}(\widetilde{q})^{4} f_{3}(\widetilde{q})^{4}}{f_{1}(\widetilde{q})^{4} f_{2}(\widetilde{q})^{4}}\right\}
\end{aligned}
$$

This can be simplified to:

$$
\frac{v^{(4)}}{32(2 \pi)^{4}} \int_{0}^{\infty} \frac{d t}{t^{3}}\left\{2 \frac{f_{4}(\widetilde{q})^{8}}{f_{1}(\widetilde{q})^{8}}+8 \frac{f_{4}(\widetilde{q})^{4} f_{3}(\widetilde{q})^{4}}{f_{1}(\widetilde{q})^{4} f_{2}(\widetilde{q})^{4}}\right\}
$$

showing that it is strictly positive. Thus the force is attractive. This is as expected, since this amplitude describes the force between $1_{f}$ and $\overline{1}_{f}$.

Finally we evaluate the last independent amplitude:

$$
\begin{aligned}
\int_{0}^{\infty} d l\left\langle\overline{\mathrm{D}} 3,+\left|e^{-l H_{c}}\right| \mathrm{D} 3,+\right\rangle= & \int_{0}^{\infty} \frac{d t}{2 t} \operatorname{tr}_{N S-R}\left(\frac{1-(-1)^{F}}{2} \frac{1-R}{2} e^{-2 t H_{0}}\right) \\
= & \frac{v^{(4)}}{32(2 \pi)^{4}} \int_{0}^{\infty} \frac{d t}{t^{3}}\left\{\frac{f_{3}(\widetilde{q})^{8}+f_{4}(\widetilde{q})^{8}-f_{2}(\widetilde{q})^{8}}{f_{1}(\widetilde{q})^{8}}\right. \\
& \left.-4 \frac{f_{4}(\widetilde{q})^{4} f_{3}(\widetilde{q})^{4}+f_{4}(\widetilde{q})^{4} f_{3}(\widetilde{q})^{4}}{f_{1}(\widetilde{q})^{4} f_{2}(\widetilde{q})^{4}}\right\}
\end{aligned}
$$

which simplifies to:

$$
\begin{aligned}
& \frac{v^{(4)}}{32(2 \pi)^{4}} \int_{0}^{\infty} \frac{d t}{t^{3}}\left\{2 \frac{f_{4}(\widetilde{q})^{8}}{f_{1}(\widetilde{q})^{8}}-8 \frac{2 f_{4}(\widetilde{q})^{4} f_{3}(\widetilde{q})^{4}}{f_{1}(\widetilde{q})^{4} f_{2}(\widetilde{q})^{4}}\right\} \\
& =\frac{v^{(4)}}{16(2 \pi)^{4}} \int_{0}^{\infty} \frac{d t}{t^{3}} \frac{f_{4}(\widetilde{q})^{8}}{f_{1}(\widetilde{q})^{8}}\left[1-4 \frac{f_{1}(\widetilde{q})^{4} f_{3}(\widetilde{q})^{4}}{f_{2}(\widetilde{q})^{4} f_{4}(\widetilde{q})^{4}}\right]
\end{aligned}
$$

Inserting the identity $f_{2}(\widetilde{q})^{2} f_{3}(\widetilde{q})^{2} f_{4}(\widetilde{q})^{2}=2$, this amplitude can be rewritten:

$$
\frac{v^{(4)}}{16(2 \pi)^{4}} \int_{0}^{\infty} \frac{d t}{t^{3}} \frac{f_{4}(\widetilde{q})^{8}}{f_{1}(\widetilde{q})^{8}}\left[1-\theta_{3}\left(\widetilde{q}^{2}\right)^{4}\right]
$$


and since $\theta_{3}>1$ for real arguments, it follows that the integrand is strictly negative, implying that the force between the $1_{f}$ and $\overline{1^{\prime}} f$ is repulsive. This confirms our claim in a previous section that the force between an adjacent suspended brane-antibrane pair is repulsive.

To summarise, we have shown that the force between two fractional $1_{f}$ branes, or between $1_{f}$ and $1_{f}^{\prime}$, is zero, as expected. The force between $1_{f}$ and $\overline{1}_{f}$ does not vanish, but is instead attractive, as one expects for a brane-antibrane pair. Finally, the force between a $1_{f}$ brane and a $\overline{1}_{f}^{\prime}$ is repulsive.

\section{Concluding Remarks}

From the variety of models considered in this paper, a number of general physical observations and open questions emerge. The fascinating structure of brane-antibrane pairs and unstable D-branes in type II string theories, and their various decay modes, are likely to be a pointer towards more fundamental structures underlying string theory. The systems we consider, with intersecting branes, lead to an even more complex picture of decay modes and interactions.

Combining some of the ingredients we have discussed could lead to dynamically stable non-BPS brane configurations and associated field theories. For example, one could imagine putting together parallel branes, which attract, and adjacent branes, which repel, in a variety of ways.

Various dual configurations exist with the Borromean property, though we have not described them here. They presumably merit careful investigation. The model that we have considered here has Bose-Fermi degeneracy in its spectrum through one-loop order. A curious occurrence of Bose-Fermi degeneracy in a very different class of non-BPS systems was described in Ref. 32, 33, and it would be interesting to know if there is any common thread that links these brane configurations and field theories.

\section{Acknowledgements:}

We would like to thank Atish Dabholkar, Keshav Dasgupta, Nick Dorey, Kimyeong Lee, Soo-Jong Rey, Ashoke Sen, Sandip Trivedi, Gerard Watts and Piljin Yi for helpful discussions. D.T. would like to express his gratitude to the Tata Institute of Fundamental Research, the Mehta Research Institute and the Korean Institute for Advanced Study for their kind hospitality. D.T. is supported by an EPSRC fellowship. 


\section{References}

[1] A. Sen, "Non-BPS States and Branes in String Theory", hep-th/9904207.

[2] E. Witten, "D-branes and K-theory", hep-th/9810188; JHEP 12 (1998) 019.

[3] P. Horava, "Type IIA D-Branes, K-Theory and Matrix Theory", hep-th/9812135; Adv. Theor. Math. Phys. 2 (1999) 1373.

[4] O. Bergman and M. Gaberdiel, "Non-BPS Dirichlet Branes", hep-th/9908126.

[5] A. Lerda and R. Russo, "Stable Non-BPS States in String Theory: A Pedagogical Review", hep-th/9905006.

[6] H. Ooguri and C. Vafa, "Two-Dimensional Black Hole and Singularities of CY Manifolds", hep-th/9511164; Nucl. Phys. B463 (1996) 55;

B. Andreas, G. Curio and D. Lüst, "The Neveu-Schwarz Five-Brane and its Dual Geometries", hep-th/9807008; JHEP 10 (1998) 022;

A. Karch, D. Lüst and D. Smith, "Equivalence of Geometric Engineering and HananyWitten via Fractional Branes", hep-th/9803232; Nucl. Phys. B533 (1998) 348.

[7] M. Douglas and G. Moore, "D Branes, Quivers and ALE Instantons", hep-th/9603167.

[8] E. Witten, "Solutions of Four Dimensional Field Theories Via M-theory", hepth/9703166; Nucl. Phys. B500 (1997) 3.

[9] A. Sen, "Supersymmetric World-Volume Action For Non-BPS D-Branes", hepth/9909062; JHEP 10 (1999) 008.

[10] A. Sen, "Universality of the Tachyon Potential", hep-th/9911116.

[11] E. Gava, K.S. Narain and M.H. Sarmadi, "On the Bound States of p-Branes and (p+2)-Branes", hep-th/9704006; Nucl. Phys. B504 (1997) 214.

[12] A. Sen, "Tachyon Condensation on the Brane Anti-Brane System", hep-th/9805170; JHEP 08 (1998) 012.

[13] P. Yi, "Membranes from Five-Branes and Fundamental Strings from Dp-Branes", hep-th/9901159; Nucl. Phys. B550 (1999) 214.

[14] A. Hanany and E. Witten, "Type IIB Superstrings, BPS Monopoles, and Threedimensional Gauge Dynamics", hep-th/9611230; Nucl. Phys. B492 (1997) 152.

[15] E. Bergshoeff and P.K. Townsend, "Solitons on the Supermembrane", hep-th/9904020; JHEP 9905 (1999) 021;

N. Lambert and D. Tong, "Kinky D-Strings", hep-th/9907098; to appear in Nucl. Phys. B.

[16] C. Kennedy and A. Wilkins, "Ramond-Ramond Couplings on Brane-Antibrane Systems", hep-th/9905195; Phys. Lett. B464 (1999) 206.

[17] A.M. Polyakov, "Quark Confinement and Topology of Gauge Groups", Nucl. Phys. B120 (1977) 429.

[18] M. Strassler, "Confining Phase of Three Dimensional Supersymmetric Quantum Electrodynamics", hep-th/9912142. 
[19] N. Manton, "Monopole Interactions at Long Range", Phys. Lett. B154 (1985) 397; (E) B157 (1985) 475;

N. Manton and G. Gibbons, "The Moduli Space for Well-Separated BPS Monopoles", hep-th/9506052; Phys. Lett. B356 (1995) 32;

K. Lee, E. Weinberg and P. Yi, "The Moduli Space of Many BPS Monopoles for Arbitrary Gauge Groups", hep-th/9602167; Phys. Rev. D54 (1996) 1633.

[20] C. Fraser and T. Hollowood, "Semi-classical Quantisation in N=4 Supersymmetric Yang-Mills Theory and Duality", hep-th/9704011; Phys. Lett. B402 (1997) 106.

[21] A. Sen, "BPS D-Branes on Nonsupersymmetric Cycles", hep-th/9812031; JHEP 12 (1998) 021.

[22] J. Majumder and A. Sen, “'Blowing Up' D-Branes on Nonsupersymmetric Cycles”, hep-th/9906109; JHEP 09 (1999) 004.

[23] J.P. Gauntlett and C.M. Hull, "BPS States With Extra Supersymmetry", hepth/9909098; JHEP 01 (2000) 004.

[24] A. Uranga, "Brane Configurations for Branes at Conifolds", hep-th/9811004; JHEP 01 (1999) 022.

[25] K. Dasgupta and S. Mukhi, "Brane Constructions, Conifolds and M-Theory", hepth/9811139; Nucl. Phys. B551 (1999) 204.

[26] G. Aldazabal and A.M. Uranga, "Tachyon Free Nonsupersymmetric Type IIB Orientifolds Via Brane-AntiBrane Systems", hep-th/9908072; JHEP 10 (1999) 024.

[27] J. Polchinski, "Tensors from K3 Orientifolds", hep-th/9606165; Phys. Rev. D55 (1997) 6423.

[28] M. Douglas, "Enhanced Gauge Symmetry in M(atrix) Theory", hep-th/9612126; JHEP 07 (1997) 004;

D.-E. Diaconescu, M. Douglas and J. Gomis, "Fractional Branes and Wrapped Branes", hep-th/9712230; JHEP 02 (1998) 013.

[29] K. Dasgupta and S. Mukhi, "Brane Constructions, Fractional Branes and Anti-de Sitter Domain Walls", hep-th/9904131; JHEP 07 (1999) 008.

[30] A. Sen, "Stable Non-BPS Bound States of BPS D-branes", hep-th/9805019; JHEP 08 (1998) 010.

[31] M. Gaberdiel and B. Stefanski, "Dirichlet Branes on Orbifolds", hep-th/9910109.

[32] M. Gaberdiel and A. Sen, "Nonsupersymmetric D-Brane Configurations with BoseFermi Degenerate Open String Spectrum", hep-th/9908060; JHEP 11 (1999) 008.

[33] M. Mihailescu, K. Oh and R. Tatar, "Non-BPS Branes on a Calabi-Yau Threefold and Bose-Fermi Degeneracy", hep-th/9910249. 